\title{
Currents in supersymmetric field theories
}

\author{
Jean-Pierre Derendinger* \\ Albert Einstein Center for Fundamental Physics \\ Institute for Theoretical Physics, Bern University \\ Sidlerstrasse 5, 3012 Bern, Switzerland \\ E-mail: derendinger@itp.unibe.ch
}

\begin{abstract}
A general formalism to construct and improve supercurrents and source or anomaly superfields in two-derivative $\mathscr{N}=1$ supersymmetric theories is presented. It includes arbitrary gauge and chiral superfields and a linear superfield coupled to gauge fields. These families of supercurrent structures are characterized by their energy-momentum tensors and $R$ currents and they display a specific relation to the dilatation current of the theory. The linear superfield is introduced in order to describe the gauge coupling as a background (or propagating) field. Supersymmetry does not constrain the dependence on this gauge coupling field of gauge kinetic terms and holomorphicity restrictions are absent. Applying these results to an effective (Wilson) description of super-YangMills theory, matching or cancellation of anomalies leads to an algebraic derivation of the allorder NSVZ $\beta$ function.
\end{abstract}

18th International Conference From the Planck Scale to the Electroweak Scale 25-29 May 2015

Ioannina, Greece

${ }^{*}$ Speaker. 


\section{Introduction: relativistic field theories}

Currents are in direct relation with the algebras of transformations acting on fields and on the action. For on-shell fields, currents verify a conservation equation giving their divergence in terms of the variation of the Lagrangian $\mathscr{L}$ under the associated field and coordinate variations. For exact symmetries, currents are then conserved, but significant information also arises from variations which are not symmetries, if the lagrangian variation is understood. Familiar examples are chiral and scale transformations which could be classical symmetries of massless field theories but are violated by calculable quantum anomalies.

Relativistic field theories have Poincaré symmetry. Fields transform linearly in a representation characterized by generators

$$
P^{\mu}=-i \partial^{\mu} \quad \text { (translations), } \quad M^{\mu v}=\Sigma^{\mu v}+i x^{\mu} \partial^{v}-i x^{v} \partial^{\mu} \quad \text { (Lorentz), }
$$

verifying the Poincaré algebra

$$
\begin{aligned}
{\left[M^{\mu v}, M^{\rho \sigma}\right] } & =-i\left(\eta^{\mu \rho} M^{v \sigma}+\eta^{v \sigma} M^{\mu \rho}-\eta^{\mu \sigma} M^{v \rho}-\eta^{v \rho} M^{\mu \sigma}\right), \\
{\left[M^{\mu \nu}, P^{\rho}\right] } & =-i\left(\eta^{\mu \rho} P^{v}-\eta^{v \rho} P^{\mu}\right), \quad\left[P^{\mu}, P^{v}\right]=0 .
\end{aligned}
$$

Hence, the Poincaré properties of fields are encoded in the choice of Lorentz generators $\Sigma_{\mu v}$. There are ten conserved currents: four translation currents assembled in the in general non-symmetric energy-momentum tensor $t_{\mu \nu}, \partial^{\mu} t_{\mu \nu}=0$ and six Lorentz currents $j_{\mu, \nu \rho}=-j_{\mu, \rho v}, \partial^{\mu} j_{\mu, \nu \rho}=0$. But Lorentz symmetry can be used to eliminate the six antisymmetric components of the energymomentum tensor, to obtain the symmetric Belinfante tensor $T_{\mu \nu}$. Lorentz currents read then

$$
j_{\rho, \mu v}=-x_{\mu} T_{\rho v}+x_{v} T_{\rho \mu}
$$

and the generators $\Sigma_{\mu \nu}$ only appear in the construction of $T_{\mu \nu}$. If the theory is coupled with diffeomorphism invariance to a background metric $g_{\mu \nu}$ or to a vierbein $e_{\mu}^{a}$, the Belinfante tensor is also obtained as

$$
T_{\mu v}=\frac{2}{e} \frac{\partial \mathscr{L}}{\partial g^{\mu \nu}}=\frac{1}{2 e}\left[\frac{\partial \mathscr{L}}{\partial e_{a}^{\mu}} e_{v a}+\frac{\partial \mathscr{L}}{\partial e_{a}^{v}} e_{\mu a}\right] .
$$

There are two relevant extensions of Poincaré space-time symmetry: firstly scale transformations (or dilatations) of fields and coordinates with algebra

$$
\left[M_{\mu v}, D\right]=0, \quad\left[D, P_{\mu}\right]=i P^{\mu} .
$$

The second relation indicates that we count scale dimensions in energy units $\left(P_{\mu}\right.$ has scale dimension +1 ). Variations are

$$
\delta x^{\mu}=-\lambda x^{\mu}, \quad \delta \phi=i \lambda D \phi, \quad D=-i x^{\mu} \partial_{\mu}-i \mathscr{D} .
$$


They are defined by assigning scale dimensions in matrix $\mathscr{D}$ (or its eigenvalues $w$ ) to fields or operators. The scale or dilatation current depends on these scale dimensions:

$$
j_{\mu}^{D}=\mathscr{V}_{\mu}+x^{v} T_{\mu \nu}
$$

in terms of the Belinfante energy-momentum tensor, with virial current

$$
\mathscr{V}_{\mu}=\frac{\partial \mathscr{L}}{\partial \partial_{\rho} \Phi}\left(\eta_{\mu \rho} \mathscr{D} \Phi+i \Sigma_{\rho \mu} \Phi\right) .
$$

Once Lorentz generators $\Sigma_{\mu \nu}$ and scale dimensions $\mathscr{D}$ have been assigned to fields, conformal boost variations with generators

$$
K^{\mu}=-i\left(2 x^{\mu} x^{v}-\eta^{\mu v} x^{2}\right) \partial_{v}-2 i x^{\mu} \mathscr{D}-2 \Sigma^{\mu v} x_{v}
$$

follow. The conformal algebra $S O(2,4) \sim S U(2,2)$ is completed by

$$
\begin{aligned}
{\left[M^{\mu v}, K^{\rho}\right] } & =-i\left(\eta^{\mu \rho} K^{v}-\eta^{v \rho} K^{\mu}\right), & & {\left[K^{\mu}, K^{v}\right]=0, } \\
{\left[P^{\mu}, K^{v}\right] } & =-2 i\left(\eta^{\mu v} D+M^{\mu v}\right), & & {\left[D, K^{\mu}\right]=-i K^{\mu} . }
\end{aligned}
$$

Since the four currents of conformal boosts (or special conformal transformations) can be expressed as

$$
K_{\rho}^{\mu}=2 x^{\mu} j_{\rho}^{D}-x^{2} T_{\rho}^{\mu}
$$

the conservation equations for the dilatation and conformal currents are

$$
\partial^{\mu} j_{\mu}^{D}=\partial^{\mu} \mathscr{V}_{\mu}+T_{\mu}^{\mu}, \quad \partial^{\rho} K_{\rho}^{\mu}=2 x^{\mu} \partial^{\rho} j_{\rho}^{D}+2 \mathscr{V}^{\mu} .
$$

In the second equation, $\mathscr{V}_{\mu}$ is the virial current (1.8) associated with the Belinfante energy-momentum tensor $T_{\mu \nu}$.

Invariance under special conformal transformations generated by (1.9) requires scale invariance. This follows already from the third commutator (1.10). Scale invariance implies full conformal symmetry if the virial current is a derivative, $\mathscr{V}_{\mu}=\partial^{v} \sigma_{\mu \nu}[1,2,3,4]$. In this case, one can replace the currents $K_{\rho}^{\mu}$ by

$$
\widehat{K}_{\rho}^{\mu}=K_{\rho}^{\mu}-2 \sigma_{\rho}^{\mu} \quad \text { with } \quad \partial^{\rho} \widehat{K}_{\rho}^{\mu}=2 x^{\mu} \partial^{\rho} j_{\rho}^{D} .
$$

Or one can improve the Belinfante energy-momentum tensor to the Callan-Coleman-Jackiw (CCJ) tensor $\Theta_{\mu v}$, to eliminate the virial current and obtain

$$
j_{\mu}^{D}=x^{v} \Theta_{\mu v}, \quad \partial^{\rho} K_{\rho}^{\mu}=2 x^{\mu} \Theta_{\rho}^{\rho} .
$$

Tracelessness of the CCJ tensor implies then conformal symmetry. In general, the first equation (1.14) defines the CCJ energy-momentum tensor. It exists if $\mathscr{V}_{\mu}=\partial^{v} \sigma_{\mu v}$. The violation or conservation of scale symmetry is measured by the trace of the energy-momentum tensor in this case only. 


\section{Supersymmetric field theories}

Supersymmetry ${ }^{1}$ extends the Poincaré algebra with spin $1 / 2$ generators $Q_{\alpha}$ and $\bar{Q}_{\dot{\alpha}}=Q_{\alpha}^{\dagger}$ :

$$
\begin{aligned}
{\left[M_{\mu \nu}, Q_{\alpha}\right] } & =-\frac{i}{4}\left(\left[\sigma_{\mu}, \bar{\sigma}_{v}\right] Q\right)_{\alpha}, & {\left[P_{\mu}, Q_{\alpha}\right] } & =0, \\
\left\{Q_{\alpha}, \bar{Q}_{\dot{\alpha}}\right\} & =2 \sigma^{\mu}{ }_{\alpha \dot{\alpha}} P_{\mu}, & \left\{Q_{\alpha}, Q_{\beta}\right\} & =0 .
\end{aligned}
$$

The corresponding conserved supercurrent $S_{\mu \alpha}, \partial^{\mu} S_{\mu \alpha}=0$ has $16_{F}-4_{F}=12_{F}$ operator components. Since currents are themselves local fields, they transform under Poincaré ( $T_{\mu \nu}$ is a twotensor, $S_{\mu \alpha}$ is a spinor-vector) and also under supersymmetry. It is natural to expect that currents assemble in supermultiplets, requiring however an equal number of bosonic and fermionic components. This cannot be achieved with the $6_{B}$ components of the symmetric energy-momentum tensor characterizing Poincaré symmetry.

Supermultiplets of $\mathscr{N}=1$ Poincaré supersymmetry are also representations of the superconformal $\mathscr{N}=1$ superalgebra $S U(2,2 \mid 1)$, with bosonic sector $S U(2,2) \times U(1)_{R} \sim S O(2,4) \times U(1)_{R}$. One simply needs to assign a scale dimension $w$ (as in the conformal case) and a $U(1)_{R}$ charge $q$ to each component field in the theory to fully define the superconformal variations. Normalizing $U(1)_{R}$ with $^{2}$

$$
\left[R, Q_{\alpha}\right]=-\frac{3}{2} i Q_{\alpha}, \quad\left[R, \bar{Q}_{\dot{\alpha}}\right]=\frac{3}{2} i \bar{Q}_{\dot{\alpha}},
$$

there are three simple rules: a chiral superfield has $w=q$, a real linear superfield ${ }^{3}$ has $w=2$, $q=0$ and of course a real superfield has $q=0$. It follows that the chiral superfield of gauge field strengths $\mathscr{W}_{\alpha}$ has $R$-charge $q=3 / 2$. Notice that $U(1)_{R}$ charge assignments can always be applied in Poincaré supersymmetry since chiral multiplet scalars live on a Kähler manifold. But $U(1)_{R}$ is not a symmetry in general, and it is not uniquely defined.

The structure of currents is as follows. Firstly, conformal invariance can be summarized in the existence of a conserved, symmetric, traceless (CCJ) energy-momentum tensor $\Theta_{\mu v}$ with $5_{B}$ fields. Secondly, $U(1)_{R}$ symmetry implies the existence of a conserved current $\mathscr{J}_{\mu}$ with $3_{B}$ fields. Thirdly, $S U(2,2 \mid 1)$ has eight supersymmetry generators. The supplementary (with respect to the Poincaré case) special supersymmetry allows to remove the " $\gamma$-trace" of the supercurrent $S_{\mu \alpha}$ : $\left(\bar{\sigma}^{\mu} S_{\mu}\right)^{\dot{\alpha}}=0$, and $8_{F}$ fields remain in the supercurrent. ${ }^{4}$ Hence, the energy-momentum tensor, the $R$-current and the supercurrent include a total of $8_{B}+8_{F}$ operators.

In 1975, Ferrara and Zumino [5] showed that in conformal Wess-Zumino and in super-YangMills (classical) theories, the currents $\Theta_{\mu \nu}$ and $S_{\mu \alpha}$ belong to a supermultiplet with an appropriate

\footnotetext{
${ }^{1}$ We consider here $\mathscr{N}=1$ supersymmetry only.

${ }^{2}$ Another convention exists in the literature, with $3 / 2$ replaced by 1 .

${ }^{3}$ See below.

${ }^{4}$ This is somewhat similar to Lorentz symmetry, used to symmetrize the energy-momentum tensor.
} 


\begin{tabular}{llll}
\hline Poincaré: & $6_{B}+12_{F}$ & $\partial^{\mu} T_{\mu v}=\partial^{\mu} S_{\mu \alpha}=0$ & $T_{\mu v}=T_{v \mu}$ \\
Conformal: & $8_{B}+8_{F}$ & $\partial^{\mu} \Theta_{\mu v}=\partial^{\mu} S_{\mu \alpha}=\partial^{\mu} \mathscr{J}_{\mu}=0$ & $\Theta_{\mu v}=\Theta_{v \mu}$ \\
& & & $\Theta^{\mu}{ }_{\mu}=\left(\bar{\sigma}^{\mu} S_{\mu}\right)^{\dot{\alpha}}=0$
\end{tabular}

Table 1: Current structure of $\mathscr{N}=1$ theories

$R$-symmetry current $\mathscr{J}_{\mu}$. The supermultiplet is cast in the real superfield

$$
J_{\mu}=\left(\bar{\sigma}_{\mu}\right)^{\dot{\alpha} \alpha} J_{\alpha \dot{\alpha}}, \quad J_{\alpha \dot{\alpha}}=\frac{1}{2}\left(\sigma^{\mu}\right)_{\alpha \dot{\alpha}} J_{\mu}
$$

submitted for on-shell fields to the supercurrent superfield equation

$$
\bar{D}^{\dot{\alpha}} J_{\alpha \dot{\alpha}}=0
$$

which includes all conservation laws and trace conditions. They also showed that breaking conformal symmetry with superpotential terms in the Wess-Zumino model introduces a specific source term in the superfield equation, $\bar{D}^{\dot{\alpha}} J_{\alpha \dot{\alpha}}=\Delta_{\alpha}$, generates values for $\Theta_{\mu}^{\mu}, \partial^{\mu} \mathscr{J}_{\mu},\left(\bar{\sigma}^{\mu} S_{\mu}\right)^{\dot{\alpha}}$ and also adds $4_{B}+4_{F}$ fields in the supercurrent superfield structure, to obtain $12_{B}+12_{F}$ fields. $^{5}$

In the superconformal case, the assignments of $R$-charges and scale dimensions are constrained by superconformal symmetry. In contrast, in Poincaré supersymmetry, these numbers are mostly arbitrary if no choice leads to scale or $R$ invariance. The $R$ and dilatation currents depend on these numbers and, since their supersymmetry partners $T_{\mu \nu}$ and $S_{\mu \alpha}$ do not depend on $q$ or $w$, the corresponding supermultiplet of currents will include a $U(1)_{R}$ current with specific $R$-charges.

\section{Supercurrent structures}

This section discusses the supercurrent superfields and equations relevant for arbitrary twoderivative $\mathscr{N}=1$ lagrangian field theories, following refs. [6,7] and also borrowing several results from ref. [8].

\subsection{The supercurrent superfield equation}

As originally shown by Ferrara and Zumino [5], the conserved supercurrent $\partial^{\mu} S_{\mu \alpha}=0$ can be embedded in a real Lorentz vector superfield $J_{\alpha \dot{\alpha}}$ submitted to a differential superfield supercurrent equation when fields solve field equations. At this point, $J_{\alpha \dot{\alpha}}$ includes $32_{B}+32_{F}$ components, or $8_{B}+8_{F}$ currents. One needs to impose a superfield differential equation to impose current

\footnotetext{
${ }^{5}$ As in the off-shell supermultiplet of minimal $\mathscr{N}=1$ supergravity.
} 
conservation and reduce the number of components. The supercurrent equation is actually of the form $^{6}$

$$
\bar{D}^{\dot{\alpha}} J_{\alpha \dot{\alpha}}=\Delta_{\alpha}, \quad D^{\alpha} J_{\alpha \dot{\alpha}}=-\bar{\Delta}_{\dot{\alpha}}
$$

which implies

$$
\overline{D D} \Delta_{\alpha}=0, \quad D^{\alpha} \Delta_{\alpha}+\bar{D}_{\dot{\alpha}} \bar{\Delta}^{\dot{\alpha}}=-2 i \partial^{\mu} J_{\mu} .
$$

The complex linear spinor superfield $\Delta_{\alpha}$ is the source of the non-conservation of (some of) the currents in $J_{\mu}$. But $\Delta_{\alpha}$ is not an arbitrary linear superfield: it should be such that $J_{\mu}$ submitted to the supercurrent equation (3.1) includes the conserved energy-momentum tensor and supercurrent required by super-Poincaré invariance of the theory.

For all supersymmetric field theories considered here, the source or anomaly superfield $\Delta_{\alpha}$ verifying this condition is of the form ${ }^{7}$

$$
\begin{array}{lll}
\Delta_{\alpha}=D_{\alpha} X+\chi_{\alpha}, & \bar{\Delta}_{\dot{\alpha}}=-\bar{D}_{\dot{\alpha}} \bar{X}+\bar{\chi}_{\dot{\alpha}}, & \bar{D}_{\dot{\alpha}} X=0, \\
\chi_{\alpha}=-\frac{1}{4} \overline{D D} D_{\alpha} U, & \bar{\chi}_{\dot{\alpha}}=\frac{1}{4} D D \bar{D}_{\dot{\alpha}} U, & U=U^{\dagger},
\end{array}
$$

which is certainly linear, $\overline{D D} \Delta_{\alpha}=0$. Then,

$$
\left\{D^{\alpha}, \bar{D}^{\dot{\alpha}}\right\} J_{\alpha \dot{\alpha}}=D^{\alpha} \Delta_{\alpha}+\bar{D}_{\dot{\alpha}} \bar{\Delta}^{\dot{\alpha}}=D D X-\overline{D D} \bar{X}
$$

since $\chi_{\alpha}$, which has the same structure as the Maxwell field strength superfield $\mathscr{W}_{\alpha}$, verifies Bianchi identity $D^{\alpha} \chi_{\alpha}=-\bar{D}_{\dot{\alpha}} \bar{\chi}^{\dot{\alpha}}$. Hence, $\chi_{\alpha}$ does not contribute to $\partial^{\mu} J_{\mu}$.

In total, superfields $J_{\alpha \dot{\alpha}}, X$ and $\chi_{\alpha}$ include $40_{B}+40_{F}$ real (or hermitian) components. Since the supercurrent superfield equation is complex linear, it imposes $2 \times\left(12_{B}+12_{F}\right)$ conditions on the $40_{B}+40_{F}$ components to leave a solution expressed in terms of $16_{B}+16_{F}$ fields.

For a given supersymmetric lagrangian, one can derive superfields $J_{\alpha \dot{\alpha}}, X$ and $\chi_{\alpha}$ (or $U$ ) verifying the supercurrent equation (3.1). These superfields are not unique, there exists supersymmetric improvement transformations acting on the conserved currents $T_{\mu \nu}$ and $S_{\mu \alpha}$ and transforming all other components of the superfields. We use the terminology supercurrent structure for each triplet of superfields $J_{\alpha \dot{\alpha}}, X$ and $\chi_{\alpha}$ submitted to the supercurrent equation $\bar{D}^{\dot{\alpha}} J_{\alpha \dot{\alpha}}=D_{\alpha} X+\chi_{\alpha}$.

The supercurrent equation (3.1) holds for solutions of the field equations only. This is similar to Noether currents associated with continuous symmetries: their expression follows from the lagrangian (they can be expressed in terms of off-shell fields) but their conservation holds for solutions of the field equations.

\footnotetext{
${ }^{6}$ The conjugate of $\bar{D}^{\dot{\alpha}} J_{\alpha \dot{\alpha}}$ is $-D^{\alpha} J_{\alpha \dot{\alpha}}$.

${ }^{7}$ These sources in the supercurrent equation are not the most general allowing conserved energy-momentum tensor and supercurrent. See also refs. $[9,10,11]$. For a long time, the literature propagated an unfortunate claim that the coexistence of $\chi_{\alpha}$ and $X$ is forbidden. The ban has been removed by Komargodski and Seiberg [8].
} 


\subsection{Component expansion}

To display the content of the supercurrent equation

$$
\bar{D}^{\dot{\alpha}} J_{\alpha \dot{\alpha}}=D_{\alpha} X+\chi_{\alpha}, \quad \bar{D}_{\dot{\alpha}} X=0, \quad \chi_{\alpha}=-\frac{1}{4} \overline{D D} D_{\alpha} U,
$$

its component form is needed. We use the following expansion of the chiral superfields $X$ and $\chi_{\alpha}$ :

$$
\begin{aligned}
X(y, \theta) & =x+\sqrt{2} \theta \psi_{X}-\theta \theta f_{X} \\
\chi_{\alpha}(y, \theta) & =-i \lambda_{\alpha}+\theta_{\alpha} D+\frac{i}{2}\left(\theta \sigma^{\mu} \bar{\sigma}^{v}\right)_{\alpha} F_{\mu v}-\theta \theta\left(\sigma^{\mu} \partial_{\mu} \bar{\lambda}\right)_{\alpha}
\end{aligned}
$$

in chiral coordinates or

$$
\begin{aligned}
X= & x+\sqrt{2} \theta \psi_{X}-\theta \theta f_{X}-i \theta \sigma^{\mu} \bar{\theta} \partial_{\mu} x-\frac{i}{\sqrt{2}} \theta \theta \bar{\theta} \bar{\sigma}^{\mu} \partial_{\mu} \psi_{X}-\frac{1}{4} \theta \theta \overline{\theta \theta} \square x, \\
\chi_{\alpha}= & -i \lambda_{\alpha}+\theta_{\alpha} D+\frac{i}{2}\left(\theta \sigma^{\mu} \bar{\sigma}^{v}\right)_{\alpha} F_{\mu v}-\theta \sigma^{\mu} \bar{\theta} \partial_{\mu} \lambda_{\alpha}-\theta \theta\left(\sigma^{\mu} \partial_{\mu} \bar{\lambda}\right)_{\alpha} \\
& -\frac{1}{2} \theta \theta\left(\sigma^{\mu} \bar{\theta}\right)_{\alpha}\left(\partial^{v} F_{v \mu}-i \partial_{\mu} D\right)+\frac{i}{4} \theta \theta \bar{\theta} \bar{\theta} \square \lambda_{\alpha}
\end{aligned}
$$

in ordinary coordinates $(x, \theta, \bar{\theta})$. For the real superfield $U$, the last eq. (3.5) implies

$$
U=\theta \sigma^{\mu} \bar{\theta} U_{\mu}+i \theta \theta \overline{\theta \lambda}+i \overline{\theta \theta} \theta \lambda+\frac{1}{2} \theta \theta \overline{\theta \theta} D+\ldots
$$

where the dots denote components of $U$ absent from $\chi_{\alpha}$ and $F_{\mu \nu}=\partial_{\mu} U_{v}-\partial_{\nu} U_{\mu}$.

With these component expansions, the resulting supercurrent superfield is ${ }^{8}$

$$
\begin{aligned}
J_{\mu}(x, \theta, \bar{\theta})= & \frac{8}{3} j_{\mu}(x)+\theta\left(S_{\mu}+2 \sqrt{2} \sigma_{\mu} \bar{\psi}_{X}\right)+\bar{\theta}\left(\bar{S}_{\mu}-2 \sqrt{2} \bar{\sigma}_{\mu} \psi_{X}\right) \\
& -2 i \theta \theta \partial_{\mu} \bar{x}+2 i \overline{\theta \theta} \partial_{\mu} x \\
& +\theta \sigma^{v} \bar{\theta}\left(8 T_{\mu \nu}-4 \eta_{\mu \nu} \operatorname{Re} f_{X}-\frac{1}{2} \varepsilon_{\mu v \rho \sigma}\left(\frac{8}{3} \partial^{\rho} j^{\sigma}-F^{\rho \sigma}\right)\right) \\
& -\frac{i}{2} \theta \theta \bar{\theta}\left(\partial_{v} S_{\mu} \sigma^{v}+2 \sqrt{2} \bar{\sigma}_{\mu} \sigma^{v} \partial_{\nu} \bar{\psi}_{X}\right) \\
& +\frac{i}{2} \bar{\theta} \theta \theta\left(\sigma^{v} \partial_{v} \bar{S}_{\mu}+2 \sqrt{2} \sigma_{\mu} \bar{\sigma}^{v} \partial_{\nu} \psi_{X}\right) \\
& -\frac{2}{3} \theta \theta \overline{\theta \theta}\left(2 \partial_{\mu} \partial^{v} j_{v}-\square j_{\mu}\right)
\end{aligned}
$$

with $T_{\mu \nu}=T_{v \mu}$. This expression solves the supercurrent equation (3.5) if $T_{\mu v}$ and $S_{\mu}$ are conserved,

$$
\partial^{\mu} T_{\mu \nu}=0, \quad \partial^{\mu} S_{\mu}=0 .
$$

Hence, $T_{\mu \nu}$ and $S_{\mu}$ will be (proportional to) the conserved energy-momentum tensor and the supercurrent. In addition, the supercurrent equation (3.5) implies the following conditions:

$$
\begin{gathered}
4 T_{\mu}^{\mu}=D+6 \operatorname{Re} f_{X}, \quad \partial^{\mu} j_{\mu}=-\frac{3}{2} \operatorname{Im} f_{X}, \\
\left(\sigma^{\mu} \bar{S}_{\mu}\right)_{\alpha}=6 \sqrt{2} \psi_{X} \alpha+2 i \lambda_{\alpha} .
\end{gathered}
$$

\footnotetext{
${ }^{8}$ In the expansion, the normalizations of $j_{\mu}$ and $T_{\mu \nu}$ have been selected to correspond to well-defined currents, see below. This has not been done for the supercurrent $S_{\mu \alpha}$ which is not explicitly used here. This expansion, originally given in ref. [8] with slightly different conventions, is not unique, see conditions (3.11).
} 
The first condition indicates that both superfields $X$ and $\chi_{\alpha}$ are sources for the trace of the energymomentum tensor. Its precise significance depends on the specific energy-momentum tensor included in $J_{\mu}$ : since the energy-momentum tensor $T_{\mu \nu}$ is defined up to improvements, the relation between the trace $T^{\mu}{ }_{\mu}$ and scale invariance or violation in the theory depends on the choice of $T_{\mu \nu}$. The second condition (3.11) indicates that $X$ only induces the nonconservation of $j_{\mu}$, which is related in general to a $R$ transformation acting in the theory. The third condition controls the violation of conformal supersymmetry. Hence, the presence of the source $\chi_{\alpha}$ breaks the correlation between $T^{\mu}{ }_{\mu}$ and $\partial^{\mu} j_{\mu}$.

The scale dimensions of the component fields are:

$$
\begin{array}{rlll}
3: & J_{\alpha \dot{\alpha}}, \quad X ; & j_{\mu}, \quad x, U_{\mu} ; \\
7 / 2: & \chi_{\alpha} ; & & S_{\mu \alpha}, \quad \psi_{X \alpha}, \lambda_{\alpha} ; \\
4: & & & T_{\mu \nu}, \quad f_{X}, D, \quad F_{\mu v} .
\end{array}
$$

To see for instance how the conservation of the energy-momentum tensor follows from the supercurrent equation, write

$$
\begin{aligned}
& J_{\mu}=\frac{8}{3} j_{\mu}+8 \theta \sigma^{v} \bar{\theta} t_{\mu v}+\theta \theta \overline{\theta \theta} d_{\mu}+\ldots \\
& t_{\mu v}=T_{\mu v}+\eta_{\mu v} t+\tau_{\mu v}, \quad T_{\mu v}=T_{v \mu}, \quad \tau_{\mu v}=-\tau_{v \mu} .
\end{aligned}
$$

The $\theta_{\alpha}$ component of the supercurrent equation, after separation of the symmetric and antisymmetric parts

$$
\theta\left\{\sigma^{\mu}, \bar{\sigma}^{v}\right\}_{\alpha}=2 \eta^{\mu v} \theta_{\alpha}, \quad \theta\left[\sigma^{\mu}, \bar{\sigma}^{v}\right]_{\alpha},
$$

and of real and imaginary parts, provides three equations: ${ }^{9}$

$$
\begin{aligned}
T_{\mu}^{\mu} & =-4 t+\frac{1}{4} D-\frac{1}{2} \operatorname{Re} f_{X}, \\
\partial^{\mu} j_{\mu} & =-\frac{3}{2} \operatorname{Im} f_{X}, \\
\varepsilon_{\mu v \rho \sigma} \tau^{\rho \sigma} & =\frac{1}{3}\left(\partial_{\mu} j_{v}-\partial_{v} j_{\mu}\right)-\frac{1}{4} F_{\mu v} .
\end{aligned}
$$

The (complex) $\theta \theta \bar{\theta}_{\dot{\alpha}}$ component gives two (real) equations:

$$
\begin{aligned}
d_{\mu} & =\partial_{\mu} \operatorname{Im} f_{X}-2 \varepsilon_{\mu v \rho \sigma} \partial^{v} \tau^{\rho \sigma}-\frac{1}{2} \partial^{v} F_{\mu v}, \\
\partial^{v} T_{\mu v} & =\frac{1}{2} \partial_{\mu}\left(2 t+T^{v}{ }_{v}-\frac{1}{4} D-\frac{1}{2} \operatorname{Re} f_{X}\right) .
\end{aligned}
$$

Since then $\partial^{v} T_{\mu \nu}=-\partial_{\mu}\left(t+\frac{1}{2} \operatorname{Re} f_{X}\right), T_{\mu \nu}$ is conserved if one defines

$$
t=-\frac{1}{2} \operatorname{Re} f_{X} .
$$

\footnotetext{
${ }^{9}$ The real (or imaginary) antisymmetric part is removed using
}

$$
\left[\sigma^{\mu}, \bar{\sigma}^{v}\right]=\frac{i}{2} \varepsilon^{\mu v \rho \sigma}\left[\sigma_{\rho}, \bar{\sigma}_{\sigma}\right] .
$$


The five equations provide then the conservation of $T_{\mu \nu}$, the expressions of components $\tau_{\mu \nu}$ and $d_{\mu}$ of $J_{\mu}$, and the two bosonic constraints (3.11).

The supercurrent superfield $J_{\mu}$ includes a conserved symmetric energy-momentum tensor $T_{\mu \nu}$ $\left(10_{B}-4_{B}=6_{B}\right)$, the conserved supercurrent $S_{\mu}\left(4 \times(4-1)_{F}=12_{F}\right)$ and a vector current $j_{\mu}$ which is not in general conserved $\left(4_{B}\right)$. Since conditions (3.11) eliminate $2_{B}+4_{F}$, the source superfields $X$ and $\chi_{\alpha}$ add $6_{B}+4_{F}$ fields, for a total of $16_{B}+16_{F}$ fields, as earlier mentioned.

Some remarks are in order. Firstly, notice that the components of the anomaly superfields $X$ and $\chi_{\alpha}$ appear in $J_{\mu}$. Hence, the symmetric part of the $\theta \sigma^{v} \bar{\theta}$ component of $J_{\mu}$ can only be identified with an energy-momentum tensor of the theory after subtraction of an anomaly contribution generated by $\operatorname{Re} f_{X}$, or by $D$, or by both, since we may as well use the first eq. (3.11) to modify the component expansion (3.9).

Secondly, even if, for a given theory, one expects to find expressions for $J_{\alpha \dot{\alpha}}, X$ and $\chi_{\alpha}$ in terms of superfields, i.e. in terms of off-shell fields, equations (3.9)-(3.11) only hold for on-shell fields. The interpretation of the components of $J_{\mu}$ in terms of currents may require the field equations. This is in particular true for the auxiliary field contributions.

\subsection{Superfield improvement transformation}

The identity

$$
2 \bar{D}^{\dot{\alpha}}\left[D_{\alpha}, \bar{D}_{\dot{\alpha}}\right] \mathscr{G}=D_{\alpha} \overline{D D} \mathscr{G}+3 \overline{D D} D_{\alpha} \mathscr{G},
$$

which holds for any superfield $\mathscr{G}$, is clearly a solution of the supercurrent superfield equation (3.5) with $J_{\alpha \dot{\alpha}}=2\left[D_{\alpha}, \bar{D}_{\dot{\alpha}}\right] \mathscr{G}, X=\overline{D D} \mathscr{G}$ and $\chi_{\alpha}=3 \overline{D D} D_{\alpha} \mathscr{G}$ (with $\mathscr{G}$ real). Hence, given superfields $J_{\alpha \dot{\alpha}}, X$ and $\chi_{\alpha}$ verifying the supercurrent equation, the transformation

$$
\begin{array}{rll}
J_{\alpha \dot{\alpha}} & \longrightarrow & \widetilde{J}_{\alpha \dot{\alpha}}=J_{\alpha \dot{\alpha}}+2\left[D_{\alpha}, \bar{D}_{\dot{\alpha}}\right] \mathscr{G}, \\
X & \longrightarrow & \widetilde{X}=X+\overline{D D} \mathscr{G} \\
\chi_{\alpha} & \longrightarrow & \tilde{\chi}_{\alpha}=\chi_{\alpha}+3 \overline{D D} D_{\alpha} \mathscr{G}
\end{array}
$$

is an ambiguity in the realization of the supercurrent superfield. The transformation necessarily involves improvement terms for $T_{\mu \nu}$ and $S_{\alpha \mu}$ : the transformed supercurrent superfield verifies again equation (3.5) and identity (3.13) holds without using any field equation. The transformed energymomentum tensor and supercurrent are then conserved and the modifications are improvements. On the other hand, the lowest component $j_{\mu}$ and the trace $T_{\mu}^{\mu}$, in particular, are non-trivially transformed.

Hence, each theory admits in principle a (continuous) family of supercurrent structures. Notice that if $\mathscr{G}$ is linear $(\overline{D D} \mathscr{G}=0), \widetilde{X}=X$. Similarly, if $\mathscr{G}=\Psi+\bar{\Psi}, \bar{D}_{\dot{\alpha}} \Psi=0$, then $\widetilde{\chi}_{\alpha}=\chi_{\alpha}$. But the 
use of transformations (3.14) may face various obstructions if conditions like gauge invariance or global definition are imposed on the supercurrent structure $J_{\alpha \dot{\alpha}}, X, \chi_{\alpha}{ }^{10}$

If the real superfield $\mathscr{G}$ of the transformation (3.14) has the expansion

$$
\begin{aligned}
\mathscr{G}= & C_{g}+i \theta \chi_{g}-i \bar{\theta} \bar{\chi}_{g}+\theta \sigma^{\mu} \bar{\theta} v_{g \mu}+\frac{i}{2} \theta \theta\left(M_{g}+i N_{g}\right)-\frac{i}{2} \overline{\theta \theta}\left(M_{g}-i N_{g}\right) \\
& +i \theta \theta \bar{\theta}\left(\bar{\lambda}_{g}+\frac{i}{2} \partial_{\mu} \chi_{g} \sigma^{\mu}\right)-i \overline{\theta \theta} \theta\left(\lambda_{g}-\frac{i}{2} \sigma^{\mu} \partial_{\mu} \bar{\chi}_{g}\right)+\frac{1}{2} \theta \theta \overline{\theta \theta}\left(D_{g}-\frac{1}{2} \square C_{g}\right),
\end{aligned}
$$

then the components of the transformed superfields $\widetilde{J}_{\mu}, \widetilde{X}$ and $\widetilde{\chi}_{\alpha}$ read

$$
\begin{aligned}
\widetilde{j}_{\mu} & =j_{\mu}-3 v_{g \mu}, & \widetilde{S}_{\mu} & =S_{\mu}+8 \sigma_{[\mu} \bar{\sigma}_{v]} \partial^{v} \chi_{g}, \\
\widetilde{\psi}_{X} & =\psi_{X}+2 \sqrt{2} i \lambda_{g}+2 \sqrt{2} \sigma^{\mu} \partial_{\mu} \bar{\chi}_{g}, & \widetilde{x} & =x+2 i\left(M_{g}-i N_{g}\right), \\
\widetilde{T}_{\mu v} & =T_{\mu v}+\left(\partial_{\mu} \partial_{v}-\eta_{\mu v} \square\right) C_{g}, & \widetilde{f}_{X} & =f_{X}+2 D_{g}-2 \square C_{g}+2 i \partial_{\mu} v_{g}^{\mu}, \\
\widetilde{F}_{\mu v} & =F_{\mu v}-24 \partial_{[\mu} v_{g v]}, & \widetilde{\lambda} & =\lambda-12 \lambda_{g}, \\
\widetilde{D} & =D-12 D_{g}, & &
\end{aligned}
$$

using the expansions (3.6) and (3.9) of $J_{\mu}, X$ and $\chi_{\alpha}$. As expected, the transformations of the energy-momentum tensor $T_{\mu \nu}$ and of the supercurrent $S_{\mu}$ are improvements.

For a given theory, each supercurrent structure is characterized either by the lowest component $j_{\mu}$ of $J_{\alpha \dot{\alpha}}$, which is a $U(1)_{R}$ current, or by the type of energy-momentum tensor it contains.

\subsection{Reductions, coupling to supergavity}

There are three simple reductions of the supercurrent structure with superfields $J_{\alpha \dot{\alpha}}, X$ and $\chi_{\alpha}$. Firstly, the Ferrara-Zumino (FZ) structure [5] with $12_{B}+12_{F}$ component fields (or operators):

$$
\text { FZ structure: } \quad \chi_{\alpha}=0, \quad \bar{D}^{\dot{\alpha}} J_{\alpha \dot{\alpha}}=D_{\alpha} X \neq 0 .
$$

Since $X \neq 0$, the $U(1)_{R}$ current $j_{\mu}$ is not conserved and the trace of the energy-momentum tensor in $J_{\alpha \dot{\alpha}}$ is correlated by supersymmetry with $\partial^{\mu} j_{\mu}$, see eqs. (3.11). Since $\chi_{\alpha}=-\frac{1}{4} \overline{D D} D_{\alpha} U$ in a generic supercurrent stucture, it can be in principle eliminated using the superfield improvement transformation (3.14) with $\mathscr{G}=\frac{1}{12} U$, to obtain a FZ structure. Problems could arise if for instance $U$ would not respect symmetries of the underlying theory. The simplest example would be a symmetry acting on $U$ with $\delta U=\mathscr{F}+\overline{\mathscr{F}}$, where $\mathscr{F}$ is a chiral function (leaving $\chi_{\alpha}$ unchanged). ${ }^{11}$ The $\mathrm{FZ}$ structure is not unique: it is preserved by improvement transformations (3.14) with $\mathscr{G}=\Psi+\bar{\Psi}$, $\bar{D}_{\dot{\alpha}} \Psi=0$.

\footnotetext{
${ }^{10}$ Although these superfields are not strictly speaking physical quantities. These conditions have been discussed in ref. [8] for some specific theories. See also ref. [6].

${ }^{11}$ Theories with Fayet-Iliopoulos terms are not problematic [6].
} 
Secondly, the $R$-invariant structure with $12_{B}+12_{F}$ component fields or operators:

$$
R \text {-invariant structure: } \quad X=0, \quad \bar{D}^{\dot{\alpha}} J_{\alpha \dot{\alpha}}=\chi_{\alpha} \neq 0 .
$$

Since $X=0$, the supercurrent superfield $J_{\alpha \dot{\alpha}}$ includes the current $j_{\mu}$ of an exact $R$-symmetry in its lowest component and the traces $T^{\mu}{ }_{\mu}$ and $\left(\bar{\sigma}^{\mu} S_{\mu}\right)^{\dot{\alpha}}$ are not zero in general. This structure can be obtained whenever $X=\overline{D D} \mathscr{U}$ for some real superfield $\mathscr{U}$. In this case $\operatorname{Im} f_{X}$ is itself the divergence of a vector field $V_{\mu}$ (off-shell) $)^{12}$ and $\partial^{\mu}\left(j_{\mu}-V_{\mu}\right)=0$. The source superfield $X$ can then be eliminated by the superfield improvement (3.14) with $\mathscr{G}=-\mathscr{U}$. The transformed $J_{\alpha \dot{\alpha}}$ has lowest component $j_{\mu}-V_{\mu}$. An obstruction can exist if $\mathscr{U}$ is not invariant under symmetries of the underlying symmetry. The $R$-invariant structure is preserved by improvement transformations (3.14) with $\mathscr{G}$ real linear $(\overline{D D} \mathscr{G}=0)$.

Thirdly, the superconformal structure with $8_{B}+8_{F}$ component fields or operators:

$$
\text { Superconformal structure: } \quad X=\chi_{\alpha}=0, \quad \bar{D}^{\dot{\alpha}} J_{\alpha \dot{\alpha}}=0 .
$$

It can be obtained whenever the source superfields in a generic structure are generated by a single real superfield $\mathscr{G}: X=\overline{D D} \mathscr{G}$ and $\chi_{\alpha}=3 \overline{D D} D_{\alpha} \mathscr{G}$. A superfield improvement (3.14) leads then to a superconformal structure with $X=\chi_{\alpha}=0$. In this case, the theory admits a conserved, symmetric and traceless symmetric energy-momentum tensor: it is conformal. In addition, it has an exact $R$-symmetry and a conserved supercurrent $S_{\mu \alpha}$ with zero $\gamma$-trace, $\left(\bar{\sigma}^{\mu} S_{\mu}\right)^{\dot{\alpha}}=0$ : the theory is superconformal. If the supersymmetric theory is coupled to conformal $\mathscr{N}=1$ supergravity, the conserved currents $j_{\mu}, T_{\mu \nu}$ and $S_{\mu \alpha}\left(8_{B}+8_{F}\right)$ couple to gauge fields of the superconformal algebra

$$
T_{\mu v} \longleftrightarrow g_{\mu v}, \quad S_{\mu \alpha} \longleftrightarrow \psi_{\mu \alpha}, \quad j_{\mu} \longleftrightarrow A_{\mu}
$$

where $\psi_{\mu \alpha}$ is the gravitino and $A_{\mu}$ the $U(1)_{R}$ gauge field.

A theory with a FZ or a $R$-invariant supercurrent structure is not superconformal. It couples to Poincaré supergravity which can be obtained by gauge-fixing a superconformal theory, using various sets of compensating fields: this procedure leads to various formulations of Poincaré supergravity characterized by their auxiliary field content $[12,13,14]$.

The chiral source multiplet $X$ of the FZ structure corresponds to the chiral compensating multiplet $S_{0}$ (with nonzero Weyl weight, usually $w=1$ and $R$-charge $q=w$ ) used in old minimal supergravity [15], with $12_{B}+12_{F}$ component fields in the off-shell Poincaré supergravity multiplet (and auxiliary fields $A_{\mu}$, with $4_{B}$ fields, and a complex scalar $\left.\left(2_{B}\right) f_{0}\right)$.

The source supermultiplet $\chi_{\alpha}$ of the $R$-invariant structure naturally couples to the real linear compensating multiplet $L_{0}(w=2)$ used in new minimal supergravity [16], with $12_{B}+12_{F}$ fields

\footnotetext{
${ }^{12}$ The condition that a vector field $V_{\mu}$ exists with $\operatorname{Im} f_{X}=\partial^{\mu} V_{\mu}$ is equivalent to the existence condition of $\mathscr{U}$, with $X=\overline{D D} \mathscr{U}$.
} 
in the off-shell Poincaré supermultiplet. The auxiliary fields are an antisymmetric tensor $B_{\mu \nu}$ with gauge invariance $\left(3_{B}\right)$ and the gauge field $A_{\mu}\left(3_{B}\right)$.

The generic structure with $X \neq 0 \neq \chi_{\alpha}$ and $16_{B}+16_{F}$ components couples finally to a conformal supergravity with both chiral and linear supermultiplets with nonzero Weyl weight. These multiplets provide the compensating fields for Poincaré gauge-fixing, supergravity auxiliary fields and $4_{B}+4_{F}$ propagating fields of the globally supersymmetric theory.

\section{Supercurrent structures of supersymmetric gauge theories}

In general, the construction of currents and of their (non-)conservation equations begins with an identity which, in essence, does not carry information. It acquires significance when field equations of a given theory are applied. In the following, we apply this method to derive supercurrent structures of generic (two-derivative) $\mathscr{N}=1$ supersymmetric theories. ${ }^{13}$

\subsection{Identities}

This subsection is purely technical. We use the following superfields:

- A set of chiral superfields $\Phi, \bar{D}_{\dot{\alpha}} \Phi=0$ and their conjugate antichiral superfields $\bar{\Phi}, D_{\alpha} \bar{\Phi}=0$. These fields are in a representation $r$, in general reducible, of the gauge group. ${ }^{14}$

- Gauge superfields: the real superfield of gauge fields $\mathscr{A}$ and the chiral superfield of gauge curvatures (field strengths) ${ }^{15}$

$$
\mathscr{W}_{\alpha}(\mathscr{A})=-\frac{1}{4} \overline{D D} e^{-\mathscr{A}} D_{\alpha} e^{\mathscr{A}}, \quad \overline{\mathscr{W}}_{\dot{\alpha}}=\frac{1}{4} D D e^{\mathscr{A}} \bar{D}_{\dot{\alpha}} e^{-\mathscr{A}}
$$

They are Lie algebra-valued, with $\mathscr{A}=\mathscr{A}^{a} T_{r}^{a}$ and generators $T_{r}^{a}$ for representation $r$, normalized with $\operatorname{Tr}\left(T_{r}^{a} T_{r}^{b}\right)=T(r) \delta^{a b}$. We will also use the real Chern-Simons superfield $\Omega$ defined by

$$
\overline{D D} \Omega=\widetilde{\operatorname{Tr}} \mathscr{W} \mathscr{W}, \quad D D \Omega=\widetilde{\operatorname{Tr}} \overline{\mathscr{W}} \mathscr{W}
$$

using the notation

$$
\widetilde{\operatorname{Tr}} \mathscr{W} \mathscr{W}=T(r)^{-1} \operatorname{Tr} \mathscr{W} \mathscr{W}
$$

\footnotetext{
${ }^{13}$ This section mostly follows refs. [6, 7].

${ }^{14}$ Component fields: complex scalars $z$, Weyl spinors $\psi$, complex auxiliary scalars $f$.

${ }^{15}$ Component fields in Wess-Zumino gauge: gauge fields $\mathscr{A}_{\mu}$ with field strengths $F_{\mu \nu}$, gauginos $\lambda$, real auxiliary scalars $D$.
} 
The gauge variation of $\Omega$ is linear, $\overline{D D} \delta \Omega=D D \delta \Omega=0$. Closed expressions for $\Omega$ are easily obtained in the abelian case:

$$
\begin{aligned}
\Omega & =-\frac{1}{4}\left[\mathscr{W}^{\alpha} D_{\alpha} \mathscr{A}+\bar{D}_{\dot{\alpha}}\left[\mathscr{A}^{\mathscr{W}^{\dot{\alpha}}}\right]\right]=-\frac{1}{4}\left[\overline{\mathscr{W}}_{\dot{\alpha}} \bar{D}^{\dot{\alpha}} \mathscr{A}+D^{\alpha}\left[\mathscr{A}_{\alpha}\right]\right] \\
& =-\frac{1}{4}\left[\mathscr{W}^{\alpha} D_{\alpha} \mathscr{A}+\overline{\mathscr{W}}_{\dot{\alpha}} \bar{D}^{\dot{\alpha}} \mathscr{A}+\frac{1}{2} \mathscr{A}\left[D^{\alpha} \mathscr{W}_{\alpha}+\bar{D}_{\dot{\alpha}} \overline{\mathscr{W}}^{\dot{\alpha}}\right]\right]
\end{aligned}
$$

The first two expresssions manifestly verify one of the two conditions (4.2), the third expression is manifestly hermitian, the equalities follow from the abelian Bianchi identity $D^{\alpha} \mathscr{W}_{\alpha}=\bar{D}_{\dot{\alpha}} \overline{\mathscr{W}}^{\dot{\alpha}}$. The non-abelian $\Omega$ is much more subtle [17].

- A linear superfield $L,{ }^{16}$ which will be used as the gauge coupling superfield. It is real with $\overline{D D} L=D D L=0$, hence the terminology linear. It will be coupled to the Chern-Simons superfield to form the gauge-invariant and real

$$
\hat{L}=L-2 \Omega
$$

with the postulate that the gauge variations of $L$ and $\Omega$ cancel in $\hat{L}: \delta L=2 \delta \Omega .{ }^{17}$

The first identity applies to an arbitrary real function $\mathscr{H}$ of the gauge-invariant superfields $\hat{L}$ and

$$
Y=\bar{\Phi} e^{\mathscr{A}} \Phi
$$

By direct calculation of, for instance, $\overline{D D} D_{\alpha}\left(\mathscr{H}-\hat{L} \mathscr{H}_{L}\right)$, one obtains ${ }^{18}$

$$
\begin{aligned}
\operatorname{Id} 1: \quad 2 \bar{D}^{\dot{\alpha}}\left[\left(\overline{\mathscr{D}}_{\dot{\alpha}} \bar{\Phi}\right) \mathscr{H}_{\Phi \Phi}\left(\mathscr{D}_{\alpha} \Phi\right)-\mathscr{H}_{L L}\left(\bar{D}_{\dot{\alpha}} \hat{L}\right)\left(D_{\alpha} \hat{L}\right)\right] \\
=-\hat{L} \overline{D D} D_{\alpha} \mathscr{H}_{L}-\left(\overline{D D} \mathscr{H}_{\Phi}\right) \mathscr{D}_{\alpha} \Phi-\overline{D D} D_{\alpha}\left(\mathscr{H}-\hat{L} \mathscr{H}_{L}\right) \\
-2 \widetilde{\operatorname{Tr}} \mathscr{W} \mathscr{W}_{\alpha} \mathscr{H}_{L}-4 \mathscr{H}_{Y} \bar{\Phi} e^{A} \mathscr{W}_{\alpha} \Phi,
\end{aligned}
$$

where subscripts indicate derivatives of $\mathscr{H}$ with respect to either $\Phi, \bar{\Phi}, \hat{L}$ or $Y$. Gauge transformations are

$$
\begin{aligned}
& \Phi \longrightarrow e^{\Lambda} \Phi, \quad \bar{\Phi} \longrightarrow \bar{\Phi} e^{\bar{\Lambda}}, \quad e^{\mathscr{A}} \longrightarrow e^{-\bar{\Lambda}} e^{\mathscr{A}} e^{-\Lambda} \\
& \mathscr{W}_{\alpha} \longrightarrow e^{\Lambda} \mathscr{W}_{\alpha} e^{-\Lambda}, \quad \overline{\mathscr{W}}_{\dot{\alpha}} \longrightarrow e^{-\bar{\Lambda} \overline{\mathscr{W}}_{\dot{\alpha}} e^{\bar{\Lambda}},}
\end{aligned}
$$

with $\Lambda=\Lambda^{a} T_{r}^{a}$ and $\bar{D}_{\dot{\alpha}} \Lambda=0$. Gauge-covariant superspace derivatives read

$$
\mathscr{D}_{\alpha} \Phi=e^{-\mathscr{A}}\left(D_{\alpha} e^{\mathscr{A}} \Phi\right), \quad \overline{\mathscr{D}}_{\dot{\alpha}} \bar{\Phi}=\left(\bar{D}_{\dot{\alpha}} \bar{\Phi} e^{\mathscr{A}}\right) e^{-\mathscr{A}}
$$

\footnotetext{
${ }^{16}$ Component fields: real scalar $C$, antisymmetric tensor $B_{\mu \nu}$ in the gauge-invariant curl $H_{\mu v \rho}=3 \partial_{[\mu} B_{v \rho]}$, spinor $\chi$.

${ }^{17}$ If the gauge group has several simple or $U(1)$ factors, we could introduce one Chern-Simons superfield $\Omega_{i}$ and one linear superfield $L_{i}$ for each factor, or define a gauge-invariant $\hat{L}=L-2 \sum_{i} c_{i} \Omega_{i}$.

${ }^{18}$ In general, the gauge invariant function $\mathscr{H}$ can depend on variables $Y_{i}$ if the representation of the chiral superfields is reducible, $r=\oplus_{i} r_{i}$. This generalization is straightforward. It may also depend on other gauge invariant quantities, such as holomorphic invariants, which we do not consider here.
} 
and

$$
\left(\overline{\mathscr{D}}_{\dot{\alpha}} \bar{\Phi}\right) e^{\mathscr{A}}\left(\mathscr{D}_{\alpha} \Phi\right)=\left(\bar{D}_{\dot{\alpha}} \bar{\Phi} e^{\mathscr{A}}\right) e^{-\mathscr{A}}\left(D_{\alpha} e^{\mathscr{A}} \Phi\right)
$$

is gauge invariant. Removing the linear superfield with $\mathscr{H}_{L}=0$ leads to

$$
2 \bar{D}^{\dot{\alpha}}\left[\left(\overline{\mathscr{D}}_{\dot{\alpha}} \bar{\Phi}\right) K_{\Phi \Phi}\left(\mathscr{D}_{\alpha} \Phi\right)\right]=-\overline{D D} D_{\alpha} K-4 K_{\Phi} \mathscr{W}_{\alpha} \Phi-\left(\overline{D D} K_{\Phi}\right)\left(\mathscr{D}_{\alpha} \Phi\right)
$$

for an arbitrary function $\mathscr{K}\left(\Phi, \bar{\Phi} e^{\mathscr{A}}\right)$. Gauge invariance reads $\mathscr{K}_{\Phi} T_{r}^{a} \Phi=\bar{\Phi} T_{r}^{a} \mathscr{K}_{\bar{\Phi}}$ for all generators.

We also need identities for gauge superfields. The tool is the non-abelian Bianchi identity:

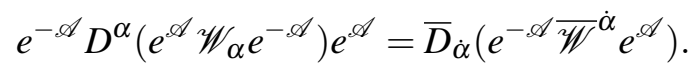

Multiplying (left) by $\mathscr{W}_{\alpha}$ and taking the trace gives

$$
\text { Id 2: } \quad \bar{D}^{\dot{\alpha}} \widetilde{T r}_{[}\left[\mathscr{W}_{\alpha} e^{-\mathscr{A}} \overline{\mathscr{W}}_{\dot{\alpha}} e^{\mathscr{A}}\right]=\widetilde{\operatorname{Tr}}\left[e^{\mathscr{A}} \mathscr{W}_{\alpha} e^{-\mathscr{A}} D^{\beta}\left(e^{\mathscr{A}} \mathscr{W}_{\beta} e^{-\mathscr{A}}\right)\right]
$$

Then, for an arbitrary (gauge-invariant) holomorphic function $F(\Phi)$,

$$
\text { Id 3: } \quad \begin{aligned}
\bar{D}^{\dot{\alpha}}\left[(F+\bar{F}) \widetilde{\operatorname{Tr}}\left[\mathscr{W}_{\alpha} e^{-\mathscr{A}} \overline{\mathscr{W}}_{\dot{\alpha}} e^{\mathscr{A}}\right]\right]= & (F+\bar{F}) \widetilde{\operatorname{Tr}}\left[e^{\mathscr{A}} \mathscr{W}_{\alpha} e^{-\mathscr{A}} D^{\beta}\left(e^{\mathscr{A}} \mathscr{W}_{\beta} e^{-\mathscr{A}}\right)\right] \\
& +\left(\bar{D}^{\dot{\alpha}} \bar{F}\right) \widetilde{\operatorname{Tr}}\left[\mathscr{W}_{\alpha} e^{-\mathscr{A}} \overline{\mathscr{W}}_{\dot{\alpha}} e^{\mathscr{A}}\right] .
\end{aligned}
$$

For given superspace lagrangians and the corresponding superfield dynamical equations, these identities "automatically" produce supercurrent structures.

\subsection{The natural supercurrent structure}

Let us consider theory

$$
\mathscr{L}=\int d^{2} \theta d^{2} \bar{\theta} \mathscr{H}(\hat{L}, Y)+\int d^{2} \theta W(\Phi)+\int d^{2} \bar{\theta} \bar{W}(\bar{\Phi}) .
$$

Gauge invariance of the holomorphic superpotential $W(\Phi)$, i.e. $W_{\Phi^{i}}\left(T_{r}^{a}\right)^{i}{ }_{j} \Phi^{j}=0$, implies $W_{\Phi} \mathscr{D}_{\alpha} \Phi$ $=D_{\alpha} W$. The $\mathscr{H}$ term in the lagrangian has in general several chiral symmetries. In particular, since $\mathscr{H}$ satisfies

$$
\mathscr{H}_{\Phi} \Phi=\bar{\Phi} \mathscr{H}_{\bar{\Phi}}=\mathscr{H}_{Y} Y,
$$

it is always invariant under the non- $R U(1)$ symmetry rotating all chiral superfields $\Phi$ by the same phase. ${ }^{19}$ Its chiral symmetries also include the $R$ symmetry (that we call $\widetilde{R}$ ) which transforms Grassmann coordinates and leaves superfields $\hat{L}$ and $\Phi$ inert. These chiral symmetries are in general broken by the superpotential.

\footnotetext{
${ }^{19}$ If the representation of the matter superfields is reducible, each irreducible component has an associated $U(1)$ global symmetry. It extends to $U(n)$ factors if the matter superfields include $n$ copies of an irreducible component.
} 
The component expansion of theory (4.13) is ${ }^{20}$

$$
\begin{aligned}
\mathscr{L}= & -\frac{1}{2} \mathscr{H}_{C C}\left[\frac{1}{2}\left(\partial_{\mu} C\right)\left(\partial^{\mu} C\right)+\frac{1}{12} H_{\mu v \rho} H^{v \mu \rho}\right]+\mathscr{H}_{z \bar{z}}\left[\left(D_{\mu} \bar{z}\right)\left(D^{\mu} z\right)+\bar{f} f\right] \\
& +\mathscr{H}_{C}\left[-\frac{1}{4} \widetilde{\operatorname{Tr}} F_{\mu v} F^{\mu v}+\frac{1}{2} \widetilde{\operatorname{Tr}} D D\right]+\frac{1}{2} \mathscr{H}_{z} D z-W_{z} f-\overline{f W}_{\bar{z}} \\
& +\frac{i}{12} \varepsilon_{\mu v \rho \sigma} H^{\mu v \rho}\left[\mathscr{H}_{C z} D^{\sigma} z-\mathscr{H}_{C \bar{z}} D^{\sigma_{\bar{z}}}\right]+\text { fermion terms }
\end{aligned}
$$

with covariant derivative $\left(D_{\mu} z\right)^{i}=\partial_{\mu} z^{i}+\frac{i}{2} A_{\mu}^{a}\left(T_{r}^{a}\right)^{i} z^{j}$ and with

$$
H_{\mu v \rho}=h_{\mu v \rho}-\omega_{\mu v \rho}
$$

in terms of the Chern-Simons form $\omega$ with normalization such that $d H=-\widetilde{\operatorname{Tr}} F \wedge F$. The kinetic metrics are then $\mathscr{H}_{z \bar{z}},-\frac{1}{2} \mathscr{H}_{C C}$ and $\mathscr{H}_{C}$ for the components of superfields $\Phi, L$ and $\mathscr{W}_{\alpha}$ respectively.

The field equations for theory (4.13) $\operatorname{are}^{21}$

$$
\begin{array}{rlrl}
L: & \overline{D D} D_{\alpha} \mathscr{H}_{L} & =0, \\
\Phi: & \overline{D D} \mathscr{H}_{\Phi} & =4 W_{\Phi}, \\
\mathscr{A}: \bar{D}^{\dot{\alpha}}\left[\mathscr{H}_{L} e^{-\mathscr{A}} \overline{\mathscr{W}}_{\dot{\alpha}} e^{\mathscr{A}}\right] & =\mathscr{W}^{\alpha} D_{\alpha} \mathscr{H}_{L}-T(r) \mathscr{H}_{Y} \Phi \bar{\Phi} e^{\mathscr{A}},
\end{array}
$$

with index $\operatorname{Tr}\left(T_{r}^{a} T_{r}^{b}\right)=T(r) \delta^{a b}$. To derive the field equation for the gauge superfield $\mathscr{A}$, it is indeed easier to use the dual chiral version of the theory, ${ }^{22}$

$$
\begin{aligned}
\mathscr{L}= & \int d^{2} \theta d^{2} \bar{\theta} \mathscr{K}(S+\bar{S}, Y) \\
& +\int d^{2} \theta\left[W(\Phi)+\frac{1}{4} S \widetilde{\operatorname{Tr}} \mathscr{W} \mathscr{W}\right]+\int d^{2} \bar{\theta}\left[\bar{W}(\bar{\Phi})+\frac{1}{4} \bar{S} \widetilde{T r}^{\overline{\mathscr{W}} \mathscr{W}}\right],
\end{aligned}
$$

where $\mathscr{K}$ is the Legendre transform of $\mathscr{H}$, and to transform the resulting field equation back into the linear version. Variation of eq. (4.18) and use of the Bianchi identity (4.10) gives then the field equation

$$
\bar{D}^{\dot{\alpha}}\left[(S+\bar{S}) e^{-\mathscr{A}} \overline{\mathscr{W}}_{\dot{\alpha}} e^{\mathscr{A}}\right]=D^{\alpha}(S+\bar{S}) \mathscr{W}_{\alpha}-2 T(r) \mathscr{K}_{Y} \Phi \bar{\Phi} e^{\mathscr{A}}
$$

Multiplying by $\mathscr{W}_{\beta}$ and taking the trace gives

$$
\bar{D}^{\dot{\alpha}}\left[(S+\bar{S}) \operatorname{Tr}\left(\mathscr{W}_{\beta} e^{-\mathscr{A}} \overline{\mathscr{W}}_{\dot{\alpha}} e^{\mathscr{A}}\right)\right]=\frac{1}{2} D_{\beta}(S+\bar{S}) \operatorname{Tr} \mathscr{W} \mathscr{W}+2 T(r) \mathscr{K}_{Y} \bar{\Phi} e^{\mathscr{A}} \mathscr{W}_{\beta} \Phi
$$

The Legendre transformation indicates then that $\mathscr{K}_{Y}=\mathscr{H}_{Y}$ and $S+\bar{S}=2 \mathscr{H}_{L}$, which in turn implies the field equation (4.17) for $\mathscr{A}$ and the relation

$$
\bar{D}^{\dot{\alpha}}\left[\mathscr{H}_{L} \operatorname{Tr}\left(\mathscr{W}_{\beta} e^{-\mathscr{A}} \overline{\mathscr{W}}_{\dot{\alpha}} e^{\mathscr{A}}\right)\right]=\frac{1}{2} D_{\beta} \mathscr{H}_{L} \operatorname{Tr} \mathscr{W} \mathscr{W}+T(r) \mathscr{H}_{Y} \bar{\Phi} e^{\mathscr{A}} \mathscr{W}_{\beta} \Phi
$$

\footnotetext{
${ }^{20}$ Gauge invariance of $\mathscr{H}$ implies $\mathscr{H}_{z} D z=\bar{z} D \mathscr{H}_{\bar{z}}$.

${ }^{21}$ We use the convention $\overline{\mathscr{W}}_{\dot{\alpha}}=\frac{1}{4} D D e^{\mathscr{A}} \bar{D}_{\dot{\alpha}} e^{-\mathscr{A}}$, with $\overline{\mathscr{W}}_{\dot{\alpha}}=-\left(\mathscr{W}_{\alpha}\right)^{\dagger}$.

${ }^{22}$ To avoid dealing with the complicated non-Abelian Chern-Simons superfield [17].
} 
With field equations (4.17) and relation (4.21), identity (4.6) immediately leads to the supercurrent structure

$$
\begin{aligned}
\bar{D}^{\dot{\alpha}} J_{\alpha \dot{\alpha}} & =D_{\alpha} X+\chi_{\alpha}, \\
J_{\alpha \dot{\alpha}} & =-2\left[\left(\overline{\mathscr{D}}_{\dot{\alpha}} \bar{\Phi}\right) \mathscr{H}_{\Phi \bar{\Phi}}\left(\mathscr{D}_{\alpha} \Phi\right)-\mathscr{H}_{L L}\left(\bar{D}_{\dot{\alpha}} \hat{L}\right)\left(D_{\alpha} \hat{L}\right)+2 \mathscr{H}_{L} \widetilde{\operatorname{Tr}}\left(\mathscr{W}_{\alpha} e^{-\mathscr{A}} \overline{\mathscr{W}}_{\dot{\alpha}} e^{\mathscr{A}}\right)\right], \\
X & =4 W \\
\chi_{\alpha} & =\overline{D D} D_{\alpha}\left(\mathscr{H}-\hat{L} \mathscr{H}_{L}\right) .
\end{aligned}
$$

This supercurrent structure can be considered as natural for theory (4.13). It actually also applies if $\mathscr{H}$ is simply a gauge-invariant function of $\hat{L}, \Phi$ and $\bar{\Phi} e^{\mathscr{A}}$, instead of a function of $\hat{L}$ and $Y$.

Using expansion (3.9) of the superfield $J_{\mu}=\left(\bar{\sigma}_{\mu}\right)^{\dot{\alpha} \alpha} J_{\alpha \dot{\alpha}}$ and also

$$
\hat{L}=C+i \theta \chi-i \bar{\theta} \bar{\chi}+\ldots, \quad \Phi=z+\sqrt{2} \theta \psi-\theta \theta f+\ldots, \quad \mathscr{W}_{\alpha}=-i \lambda_{\alpha}+\ldots
$$

the lowest component of the supercurrent superfield (4.22) is

$$
\left.j_{\mu}^{\widetilde{R}} \equiv \frac{3}{8}\left(\bar{\sigma}_{\mu}\right)^{\dot{\alpha} \alpha} J_{\alpha \dot{\alpha}}\right|_{\theta=0}=-\frac{3}{2} \mathscr{H}_{z \bar{z}} \psi \sigma_{\mu} \bar{\psi}+\frac{3}{4} \mathscr{H}_{C C} \chi \sigma_{\mu} \bar{\chi}+\frac{3}{2} \mathscr{H}_{C} \widetilde{\operatorname{Tr}} \lambda \sigma_{\mu} \bar{\lambda}
$$

It is the Noether current of $\widetilde{R}$-transformations with chiral charges $-3 / 2,-3 / 2$ and $3 / 2$ for $\chi, \psi$ and $\lambda$ respectively. The chiral charges of superfields $\Phi, L$ and $\mathscr{W}_{\alpha}$ for this $U(1)_{\widetilde{R}}$ are then $q=0$, $0,3 / 2$ in this supercurrent structure and $U(1)_{\widetilde{R}}$ only acts on the Grassmann coordinates. ${ }^{23}$ It is an automatic symmetry of $D$-term lagrangians and, according to the second eq. (3.11), the $\widetilde{R}$ current is conserved if the superpotential vanishes, $\partial^{\mu} j_{\mu}^{\widetilde{R}}=-\frac{3}{2} \operatorname{Im} f_{X}$.

The supercurrent superfield $J_{\alpha \dot{\alpha}}$ of eqs. (4.22) also contains the Belinfante (symmetric, gaugeinvariant) energy-momentum tensor $T_{\mu \nu}$ for theory (4.13). Omitting fermions and gauge fields, its expression is

$$
\begin{aligned}
T_{\mu \nu}= & -\frac{1}{2} \mathscr{H}_{C C}\left(\partial_{\mu} C\right)\left(\partial_{\nu} C\right)-\frac{1}{4} \mathscr{H}_{C C} h_{\mu \rho \sigma} h_{v}{ }^{\rho \sigma}+\mathscr{H}_{z \bar{z}}\left[\left(\partial_{\mu} z\right)\left(\partial_{v} \bar{z}\right)+\left(\partial_{v} z\right)\left(\partial_{\mu} \bar{z}\right)\right] \\
& -\eta_{\mu \nu}\left(-\frac{1}{4} \mathscr{H}_{C C}\left(\partial_{\rho} C\right)\left(\partial^{\rho} C\right)-\frac{1}{24} \mathscr{H}_{C C} h_{\rho \sigma \lambda} h^{\rho \sigma \lambda}+\mathscr{H}_{z \bar{z}}\left[\left(\partial_{\rho} z\right)\left(\partial^{\rho} \bar{z}\right)+\bar{f} f\right]\right) \\
& +\frac{1}{2} \eta_{\mu \nu} \mathscr{H}_{C} \widetilde{\operatorname{Tr}}\left(D^{2}\right)+\frac{1}{2} \eta_{\mu \nu} \operatorname{Re} f_{X},
\end{aligned}
$$

with auxiliary fields ${ }^{24}$

$$
f_{X}=4 W_{z} f, \quad \bar{f} \mathscr{H}_{\bar{z} z}=W_{z}, \quad D^{a}=-\frac{1}{2} \mathscr{H}_{C}^{-1} \mathscr{H}_{z} T_{r}^{a} z=-\frac{1}{2} \mathscr{H}_{C}^{-1} \mathscr{H}_{Y} \bar{z} T_{r}^{a} z .
$$

\footnotetext{
${ }^{23}$ The charge $q=3 / 2$ of $\mathscr{W}_{\alpha}$ is due to the derivatives in $\mathscr{W}_{\alpha}=-\frac{1}{4} \overline{D D} e^{-\mathscr{A}} D_{\alpha} e^{\mathscr{A}}$.

${ }^{24}$ The auxiliary field contribution to $T_{\mu \nu}$ is $\eta_{\mu \nu} V$, where $V$ is the usual scalar potential

$$
V(C, z, \bar{z})=\frac{1}{2} \mathscr{H}_{C} \widetilde{\mathrm{Tr}} D^{2}+\mathscr{H}_{\bar{z} z} \bar{f} f .
$$
}


Notice that terms depending on $\mathscr{H}_{C z}$ or $\mathscr{H}_{C z}$ present in the lagrangian do not appear in the Belinfante tensor $T_{\mu \nu}$. If the superpotential vanishes, $f=f_{X}=0$.

Hence, the Belinfante tensor and the $\widetilde{R}$ current with zero charge chiral superfields are partners in the natural supercurrent structure.

\subsection{The improved supercurrent structure}

Suppose that we assign scale dimensions $w$ and $R$-charges $q$ to the chiral superfields $\Phi .{ }^{25}$ The behaviour of theory (4.13) under dilatations is controlled by two superfields:

$$
\begin{aligned}
& \Delta_{(w)}=w \Phi \mathscr{H}_{\Phi}+w \Phi \mathscr{H}_{\Phi}+2 \hat{L} \mathscr{H}_{\hat{L}}-2 \mathscr{H} \quad \text { (real), } \\
& \widetilde{\Delta}_{(w)}=w \Phi W_{\Phi}-3 W \quad \text { (chiral). }
\end{aligned}
$$

Scale invariance is obtained if $\Delta_{(w)}=\widetilde{\Delta}_{(w)}=0$ for some $w$. Similarly, the variation under $R$ is controlled by

$$
\begin{array}{ll}
\Xi_{(q)}=i q\left(\Phi \mathscr{H}_{\Phi}-\bar{\Phi} \mathscr{H}_{\Phi}\right) & \text { (real), } \\
\widetilde{\Xi}_{(q)}=q \Phi W_{\Phi}-3 W=\widetilde{\Delta}_{(q)} & \text { (chiral). }
\end{array}
$$

The $R$-current (4.23) indicates that chiral superfields in the natural structure (4.22) have zero charge, $\Xi_{(0)}=0$, and the source superfields of this structure are then

$$
X=-\frac{4}{3} \widetilde{\Delta}_{(0)}=-\frac{4}{3} \widetilde{\Xi}_{(0)}, \quad \chi_{\alpha}=-\frac{1}{2} \overline{D D} D_{\alpha} \Delta_{(0)} .
$$

From theory (4.13), one easily deduces the dilatation current, expressed in terms of the Belinfante tensor, and its divergence (using field equations). There is of course a virial current for the scalar fields,

$$
j_{\mu}^{D}=-\frac{1}{2}\left[\left.\frac{\partial}{\partial C} \Delta_{(0)}\right|_{\theta=0} \partial_{\mu} C\right]+x^{v} T_{\mu \nu}
$$

and it is not a derivative in general: the linear superfield coupled to chiral fields opposes the existence of the CCJ tensor. But the virial current also cancels with scale invariance condition $\Delta_{(0)}=0$. For the natural structure, $R$-charges and scale dimensions of $\Phi$ vanish. We now wish to obtain supercurrent structures for nonzero weights of $\Phi$.

Applying to the natural structure (4.22) the superfield improvement transformation (3.14) with

$$
\mathscr{G}=-\frac{w}{6}\left(\mathscr{H}_{\Phi} \Phi+\bar{\Phi} \mathscr{H}_{\Phi}\right)
$$

the chiral source superfield $X$ becomes

$$
\widetilde{X}=-\frac{4}{3} \widetilde{\Delta}_{(w)}+\frac{4}{3} w W_{\Phi} \Phi-\frac{w}{6} \overline{D D}\left(\mathscr{H}_{\Phi} \Phi+\bar{\Phi} \mathscr{H}_{\Phi}\right)
$$

\footnotetext{
${ }^{25}$ We suppress indices. The introduction of independent $w_{i}$ and $q_{i}$ for each irreducible component $\Phi_{i}$ is straightforward. The scale dimension of $\hat{L}$ is always $w=2$ : the dimension of $\Omega$ is canonical. The linear $L$ contains a dimensionthree vector field $\varepsilon_{\mu v \rho \sigma} \partial^{v} b^{\rho \sigma}$ which is transverse, $\partial^{\mu} v_{\mu}=0$.
} 
or

$$
\widetilde{X}=-\frac{4}{3} \widetilde{\Delta}_{(w)}+\frac{w}{6} \overline{D D}\left(\mathscr{H}_{\Phi} \Phi-\bar{\Phi} \mathscr{H}_{\bar{\Phi}}\right)=-\frac{4}{3} \widetilde{\Delta}_{(w)}-\frac{i}{6} \overline{D D} \Xi_{(w)}
$$

using the field equation of $\Phi$. The resulting improved supercurrent structure is then

$$
\begin{aligned}
\bar{D}^{\dot{\alpha}} \widetilde{J}_{\alpha \dot{\alpha}} & =D_{\alpha} \widetilde{X}+\widetilde{\chi}_{\alpha}, \\
\widetilde{J}_{\alpha \dot{\alpha}} & =-2\left[\left(\overline{\mathscr{D}}_{\dot{\alpha}} \bar{\Phi}\right) \mathscr{H}_{\Phi \Phi}\left(\mathscr{D}_{\alpha} \Phi\right)-\mathscr{H}_{L L}\left(\bar{D}_{\dot{\alpha}} \hat{L}\right)\left(D_{\alpha} \hat{L}\right)+2 \mathscr{H}_{L} \widetilde{T}\left(\mathscr{W}_{\alpha} e^{\left.\left.-\mathscr{A} \bar{W}_{\dot{\alpha}} e^{\mathscr{A}}\right)\right]}\right.\right. \\
& \quad-\frac{w}{3}\left[D_{\alpha}, \bar{D}_{\dot{\alpha}}\right]\left(\mathscr{H}_{\Phi} \Phi+\bar{\Phi} \mathscr{H}_{\bar{\Phi}}\right), \\
\widetilde{X} & =-\frac{4}{3} \widetilde{\Delta}_{(w)}+\frac{w}{6} \overline{D D}\left(\mathscr{H}_{\Phi} \Phi-\bar{\Phi} \mathscr{H}_{\bar{\Phi}}\right), \\
\tilde{\chi}_{\alpha} & =-\frac{1}{2} \overline{D D} D_{\alpha} \Delta_{(w)} .
\end{aligned}
$$

In the canonical Wess-Zumino model, $\mathscr{H}=\bar{\Phi} \Phi$, the supercurrent superfield reduces to

$$
\widetilde{J}_{\alpha \dot{\alpha}}=\frac{4}{3}\left[\left(w-\frac{3}{2}\right)\left(\bar{D}_{\dot{\alpha}} \bar{\Phi}\right)\left(D_{\alpha} \Phi\right)-i w\left(\sigma^{\mu}\right)_{\alpha \dot{\alpha}} \bar{\Phi} \overleftrightarrow{\partial_{\mu}} \Phi\right]
$$

with $R$-current

$$
j_{\mu}=\left(w-\frac{3}{2}\right) \psi \sigma_{\mu} \bar{\psi}-i w \bar{z} \overleftrightarrow{\partial}_{\mu} z
$$

two results often used in the literature with canonical scale dimension or $R$-charge $w=1$.

As required for superconformal invariance, the source superfields in structure (4.32) vanish if two conditions are fulfilled. Firstly, scale invariance $\Delta_{(w)}=\widetilde{\Delta}_{(w)}=0$ and secondly that the theory has a $U(1) R$-symmetry rotating $\Phi$ with charges $q=w: w\left(\mathscr{H}_{\Phi} \Phi-\bar{\Phi} \mathscr{H}_{\bar{\Phi}}\right)=0$. This second condition is certainly fulfilled if $\mathscr{H}$ is a fonction of $\hat{L}$ and $Y$. If it is not verified, scale invariance may not imply conformal invariance. A simple example is the Kähler potential $K=\frac{1}{2}\left(\Phi^{2} \bar{\Phi}+\bar{\Phi}^{2} \Phi\right)$ for a single chiral superfield: the CCJ energy-momentum tensor does not exist and scale invariance with $w=2 / 3$ does not imply conformal invariance. In $\widehat{J}_{\alpha \dot{\alpha}}$, the energy-momentum tensor $\Theta_{\mu v}$ is related to the Belinfante tensor by the improvement

$$
\begin{aligned}
\Theta_{\mu v} & =T_{\mu v}-\frac{1}{6}\left(\partial_{\mu} \partial_{v}-\eta_{\mu v} \square\right) w\left(\mathscr{H}_{z} z+\bar{z} \mathscr{H}_{\bar{z}}\right) \\
& =T_{\mu v}-\frac{1}{3}\left(\partial_{\mu} \partial_{v}-\eta_{\mu v} \square\right) w \mathscr{H}_{y} y, \quad y=\bar{z} z .
\end{aligned}
$$

The virial current derived from the difference between the divergence of the dilatation current, which is not in the supercurrent structure, and $\Theta^{\mu}{ }_{\mu}$ is

$$
\widehat{\mathscr{V}}_{\mu}=-\frac{1}{2} \frac{\partial \Delta_{(w)}}{\partial C} \partial_{\mu} C
$$

It vanishes if $\Delta_{(w)}=0$, i.e. if $\mathscr{H}$ has scale dimension two for scale dimensions $w$. Requiring the existence of the CCJ energy-momentum tensor selects a particular class of functions $\mathscr{H}$ where $\partial_{\hat{L}} \Delta_{(w)}$ is a function of $\hat{L}$ only, for a choice of $w$ :

$$
\mathscr{H}(\hat{L}, Y)=\mathscr{F}_{1}(\hat{L})+\mathscr{F}_{2}(Y)+\mathscr{I}(\hat{L}, Y), \quad w Y \mathscr{I}_{Y}+\hat{L} \mathscr{I}_{L}=\mathscr{I}
$$


The second equation indicates that the coupling of chiral to matter multiplets should have scale dimension two, and the corresponding interaction terms should be scale invariant. For instance,

$$
\mathscr{I}(\hat{L}, Y)=\hat{L} \widetilde{\mathscr{I}}(X), \quad X=Y \hat{L}^{-w}
$$

allows to find an energy-momentum tensor such that $\partial^{\mu} j_{\mu}^{D}=\Theta^{\mu}{ }_{\mu}$.

The supercurrent superfield $\widehat{J}_{\alpha \dot{\alpha}}$ includes in its lowest component the current of the $R$ transformation with $R$ charges 0 and $w$ for $\hat{L}$ and $\Phi$ respectively. Gauginos, fermions $\psi$ in $\Phi$ and $\chi$ in $L$ have chiral weights $3 / 2, w-3 / 2$ and $-3 / 2$ respectively. Notice that $w$ has been originally introduced as the scale dimension of $\Phi$ and it here also plays the role of an $R$ charge. This is reminiscent of the chirality condition in a superconformal theory, in which the scale dimension and the $U(1)_{R}$ charge are identified. This $R$ transformation combines $\widetilde{R}$ and a $U(1)_{\mathscr{Z}}$ non- $R$ transformation acting on $\Phi$ with charge $w$. The non-conservation equation for the vector superfield $\mathscr{Z}$ of $U(1)_{\mathscr{Z}}$ is

$$
\overline{D D} \mathscr{Z}=4 w W_{\Phi} \Phi-\frac{1}{2} \overline{D D}\left(w \mathscr{H}_{\Phi} \Phi-w \bar{\Phi} \mathscr{H}_{\Phi}\right), \quad \quad \mathscr{Z}=\frac{1}{2}\left(w \mathscr{H}_{\Phi} \Phi+w \bar{\Phi} \mathscr{H}_{\Phi}\right) .
$$

Acting with $D_{\alpha}$, using identity (3.13) and the field equations immediately leads to the improvement transformation (4.29) applied to the natural structure.

We may further improve the structure (4.32) to a Ferrara-Zumino supercurrent with $\chi_{\alpha}=0$. This second improvement would lead to a supercurrent depending on the superfield $\Delta_{(w)}$,

$$
\widehat{J}_{\alpha \dot{\alpha}} \longrightarrow \widehat{J}_{\alpha \dot{\alpha}}+\frac{1}{3}\left[D_{\alpha}, \bar{D}_{\dot{\alpha}}\right] \Delta_{(w)} .
$$

The content of the supercurrent structure (4.32) is however more intuitive, with the lagrangian superfield $\mathscr{H}$ defining the supercurrent superfield $\widehat{J}_{\alpha \dot{\alpha}}$ and the scale- and $R$-breaking superfields $\Delta_{(w)}, \widetilde{\Delta}_{(w)}$ and $\Xi_{(w)}$ defining the source superfields $\widehat{X}$ and $\widehat{\chi}_{\alpha}$.

\section{Anomalies and super-Yang-Mills theory}

Consider now super-Yang-Mills theory described by an effective Wilson lagrangian $\mathscr{L}_{W, \mu}$. This local lagrangian is obtained schematically by functional integration of the super-Yang-Mills lagrangian $\mathscr{L}_{S Y M}$ with a low-energy cutoff $\mu$ kept in the perturbative regime. ${ }^{26}$ With the linear superfield used as gauge-coupling field, we have two gauge-invariant superfields, $\hat{L}$ and $\widetilde{\operatorname{Tr}} \mathscr{W} \mathscr{W}$. In principle, at two-derivative level,

$$
\mathscr{L}_{\mu}=\int d^{2} \theta d^{2} \bar{\theta} \mathscr{H}(\hat{L})+\int d^{2} \theta W(\widetilde{\operatorname{Tr}} \mathscr{W} \mathscr{W})+\text { h.c. }
$$

\footnotetext{
${ }^{26}$ The lagrangian $\mathscr{L}_{W, \mu}$ is then used to calculate amplitudes with $\mu$ as UV cutoff.
} 
Omitting fermionic terms ${ }^{27}$ generated by higher-order terms in $\widetilde{\operatorname{Tr}} \mathscr{W} \mathscr{W}$, the superpotential reduces to

$$
\frac{A}{4} \int d^{2} \theta \widetilde{\operatorname{Tr}} \mathscr{W} \mathscr{W}=\frac{A}{2} \int d^{2} \theta d^{2} \bar{\theta} \hat{L}
$$

up to a derivative $\partial_{\mu}(\ldots)$ and it can be absorbed in $\mathscr{H}$. At two derivatives then, $\mathscr{H}$ only depends on $\hat{L}$.

We next identify the scalar $C=\left.\hat{L}\right|_{\theta=0}$ with the gauge coupling defined at some arbitrary scale $M$ :

$$
C=m^{2} g^{2}(M)
$$

where the irrelevant mass scale $m$ keeps track of the mass dimension two of $C .{ }^{28}$ As a consequence, we use

$$
\mathscr{L}_{W, \mu}=m^{2} \int d^{2} \theta d^{2} \bar{\theta} \mathscr{H}\left(\frac{\hat{L}}{m^{2}}\right)=m^{2} \mathscr{H}_{C} \mathscr{L}_{S Y M}+\ldots
$$

and the natural supercurrent structure (4.22) reduces to

$$
\begin{aligned}
\bar{D}^{\dot{\alpha}} J_{\alpha \dot{\alpha}} & =D_{\alpha} X+\chi_{\alpha}, \\
J_{\alpha \dot{\alpha}} & =-4 m^{2} \mathscr{H}_{L} \widetilde{\operatorname{Tr}}\left(\mathscr{W}_{\alpha} e^{-\mathscr{A}} \overline{\mathscr{W}}_{\dot{\alpha}} e^{\mathscr{A}}\right)+2 m^{2} \mathscr{H}_{L L}\left(\bar{D}_{\dot{\alpha}} \hat{L}\right)\left(D_{\alpha} \hat{L}\right), \\
X & =0, \quad \chi_{\alpha}=m^{2} \overline{D D} D_{\alpha}\left(\mathscr{H}-\hat{L} \mathscr{H}_{L}\right) .
\end{aligned}
$$

It includes the Belinfante energy-momentum tensor and the $U(1)_{\widetilde{R}}$ current (4.23).

A perturbative expansion of the Wilson gauge coupling

$$
\frac{1}{g_{W}^{2}(\mu)}=m^{2} \frac{\partial \mathscr{H}}{\partial C}=m^{2} \mathscr{H}_{C}
$$

would indicate

$$
\mathscr{H}=\ln \hat{L}+\sum_{k \geq 1} \frac{c_{k}}{k}\left[\frac{\hat{L}}{m^{2}}\right]^{k}
$$

where the numbers $c_{k}$ are the $k$-loop corrections which depend on $\mu / M$ and on the physical coupling $g^{2}(M)$. Notice that with this expansion, the quantum corrections to $\chi_{\alpha}$ appear at two loops. This however holds under the assumption that the quantum correction in $\mathscr{H}$ admits a power expansion around $g^{2}=0$ and this is not what we will find. We are interested in a derivation of $\mathscr{H}$ to all orders using two arguments. Firstly, it is known that the $\mu$-dependence of the Wilson coupling stops at one-loop $[18,19]$ :

$$
\mu \frac{d}{d \mu} \frac{1}{g_{W}^{2}}=\frac{b_{0}}{8 \pi^{2}}
$$

\footnotetext{
${ }^{27}$ Supersymmetric contributions which vanish in a bosonic backgound.

${ }^{28}$ Scale transformations act on $C$ and not on $m$. But $\mu$ and $M$ are actually energy or momentum scales on which scale transformations act.
} 
where $b_{0}=3 C(G)$ is the coefficient of the one-loop $\beta$ function. ${ }^{29}$ This indicates that

$$
\begin{aligned}
\mathscr{H} & =\ln \hat{L}+\frac{3 C(G)}{8 \pi^{2}} \ln \frac{\mu}{M} \frac{\hat{L}}{m^{2}}+\mathscr{H}_{\text {pert. }}\left(\hat{L} / m^{2}\right), \\
\frac{1}{g_{W}^{2}(\mu)} & =\frac{1}{g_{W}^{2}(M)}+\frac{3 C(G)}{8 \pi^{2}} \ln \frac{\mu}{M}, \quad \frac{1}{g_{W}^{2}(M)}=\frac{m^{2}}{C}+m^{2} \frac{\partial}{\partial C} \mathscr{H}_{\text {pert. }}\left(\hat{L} / m^{2}\right),
\end{aligned}
$$

where the quantum correction $\mathscr{H}_{\text {pert }}$. does not depend on $\mu$. Secondly anomaly-matching in the Wilson effective lagrangian. The outcome will be an algebraic derivation, from anomalies, of the all-order $\beta$ function for super-Yang-Mills theory originally obtained by Novikov, Shifman, Vainshtein and Zakharov (NSVZ) [20] from instanton calculations of the gaugino condensate and by Jones [21] using Ward identity arguments.

Quantum anomalies affect the chiral $\widetilde{R}$-current and the trace of the energy-momentum tensor, or the dilatation current. The $U(1)_{\widetilde{R}}$ transformation of the gaugino $\lambda_{\alpha}$ with charge $q=3 / 2$ induces a one-loop chiral $\widetilde{R}$-gauge-gauge mixed anomaly: ${ }^{30}$

$$
\partial^{\mu} j_{\mu}^{(\lambda)}=\frac{1}{16 \pi^{2}} \frac{3}{2} C(G) \widetilde{\operatorname{Tr}} F^{\mu v} \widetilde{F}_{\mu v}+\ldots, \quad j_{\mu}^{(\lambda)}=\frac{3}{2} \frac{1}{g_{W}^{2}(\mu)} \lambda \sigma^{\mu} \bar{\lambda}
$$

Since $\partial^{\mu} j_{\mu}=-\frac{3}{2} \operatorname{Im} f_{X}$ in the supercurrent structure, the anomaly adds a quantum correction to the chiral source superfield

$$
X_{(\text {anomaly })}=-\frac{1}{8 \pi^{2}} C(G) \widetilde{\operatorname{Tr}} \mathscr{W} \mathscr{W}
$$

Comparing with $\widetilde{X}$ in (4.32), we can write

$$
X_{(\text {anomaly })}=-\frac{4}{3} \widetilde{\Delta}_{(\text {anomaly })}, \quad \widetilde{\Delta}_{(\text {anomaly })}=\frac{3}{32 \pi^{2}} C(G) \widetilde{\operatorname{Tr}} \mathscr{W} \mathscr{W} .
$$

And using the definition (4.25), the anomaly could be generated in an effective lagrangian with the $F$-term superpotential

$$
W_{(\text {anomaly })}=\frac{1}{32 \pi^{2}} C(G) \widetilde{\operatorname{Tr}} \mathscr{W} \mathscr{W}[\ln \widetilde{\operatorname{Tr}} \mathscr{W} \mathscr{W}-1]
$$

since $\widetilde{\operatorname{Tr}} \mathscr{W} \mathscr{W}$ has $\widetilde{R}$-charge and scale dimension three. ${ }^{31}$ At the perturbative level, as earlier indicated, the superpotential (5.13) is fermionic. When gauginos condensate, it gives rise to the Veneziano-Yankielowicz superpotential [22]. Under a scale or $\widetilde{R}$ transformation with parameters $\beta$ and $\alpha$,

$$
\delta \int d^{4} x \int d^{2} \theta W_{(\text {anomaly })}+\text { h.c. }=\frac{3 C(G)}{32 \pi^{2}}(\beta+i \alpha) \int d^{4} x \int d^{2} \theta \widetilde{\operatorname{Tr}} \mathscr{W} \mathscr{W}+\text { h.c. }
$$

\footnotetext{
${ }^{29}$ And $C(G)$ is the quadratic Casimir $C(G) \delta^{a b}=f^{a c d} f^{b c d}$ in terms of structure constants.

${ }^{30}$ Dots indicate terms needed by supersymmetry.

${ }^{31} \mathrm{Up}$ to an arbitrary term linear in $\widetilde{\mathrm{T}} \mathscr{W}^{\mathscr{W}}$ which would set its scale.
} 
This is precisely the variation induced by a formal rescaling $\mu \rightarrow e^{\beta+i \alpha} \mu$ of the Wilson scale in the lagrangian defined by expression (5.9). In this sense, the one-loop correction to $\mathscr{L}_{W, \mu}$ is a one-loop anomaly-matching term.

Supersymmetry relates the contributions of $X_{(\text {anomaly })}$ to $\partial^{\mu} j_{\mu}$ and to $T_{\mu}^{\mu}$ :

$$
\partial^{\mu} j_{\mu}=\frac{3 C(G)}{32 \pi^{2}} \widetilde{\operatorname{Tr}} F^{\mu v} \widetilde{F}_{\mu \nu}+\ldots \quad \longleftrightarrow \quad T_{\mu}^{\mu}=\frac{3 C(G)}{8 \pi^{2}} \mathscr{L}_{S Y M}+\ldots
$$

but the last equation is not the result predicted by the dilatation anomaly of a gaugino with scale dimension $3 / 2,{ }^{32}$

$$
T^{\mu}{ }_{\mu}=\frac{1}{12 \pi^{2}} \frac{3}{2} C(G) \mathscr{L}_{S Y M}+\ldots
$$

Hence, there is a residual anomaly

$$
T_{\mu}^{\mu}-\frac{3 C(G)}{8 \pi^{2}} \mathscr{L}_{S Y M}=-\frac{C(G)}{4 \pi^{2}} \mathscr{L}_{S Y M}+\ldots
$$

This residual anomaly must be compensated in the Wilson effective lagrangian by renormalizationgroup invariance of the theory, in terms of the dependence on $M$ or $g^{2}(M)$ or $C$.

We can now use the real $\hat{L}$ and the source superfield $\chi_{(\text {anomaly) } \alpha}$ for this cancellation mechanism. We need

$$
\chi_{(\text {anomaly }) \alpha}=-\frac{C(G)}{8 \pi^{2}} \overline{D D} D_{\alpha} \hat{L}
$$

The last eq. (5.5) indicates that this contribution is generated by the anomaly counterterm

$$
\mathscr{H}_{(\text {anomaly })}=\frac{C(G)}{8 \pi^{2}} \frac{\hat{L}}{m^{2}}\left[\ln \frac{\hat{L}}{m^{2}}-1\right],
$$

up to an arbitrary invariant linear term. ${ }^{33}$ This counterterm is the all-order correction $\mathscr{H}_{\text {pert. }}$ in the effective lagrangian which is then defined by

$$
m^{2} \mathscr{H}=m^{2} \ln \hat{L}+\frac{3 C(G)}{8 \pi^{2}} \ln \frac{\mu}{M} \hat{L}+\frac{C(G)}{8 \pi^{2}}\left[\hat{L} \ln \frac{\hat{L}}{m^{2}}-\hat{L}\right] .
$$

Hence, the presence of the gauge coupling superfield $L$ takes care of the different values of the chiral $\widetilde{R}$ and $T^{\mu}{ }_{\mu}$ anomalies, and produces the all-order correction $\mathscr{H}_{\text {pert. }}$. In the lagrangian defined by eq. (5.20), the Wilson gauge coupling is

$$
\begin{aligned}
\frac{1}{g_{W}^{2}(\mu)} & =\frac{m^{2}}{C}+\frac{3 C(G)}{8 \pi^{2}} \ln \frac{\mu}{M}+\frac{C(G)}{8 \pi^{2}} \ln \frac{C}{m^{2}} \\
& =\frac{1}{g^{2}(M)}+\frac{3 C(G)}{8 \pi^{2}} \ln \frac{\mu}{M}+\frac{C(G)}{8 \pi^{2}} \ln g^{2}(M), \\
\frac{1}{g_{W}^{2}(M)} & =\frac{1}{g^{2}(M)}+\frac{C(G)}{8 \pi^{2}} \ln g^{2}(M) .
\end{aligned}
$$

\footnotetext{
${ }^{32}$ Gauginos are superpartners of gauge fields, their anomalous dimensions vanish.

${ }^{33}$ Which is a one-loop term, see eq. (5.7).
} 
Finally, since the reference energy scale $M$ is arbitrary, the renormalization-group equation

$$
M \frac{d}{d M} g_{W}^{2}(\mu)=0
$$

leads to the $\beta$ function

$$
\beta\left(g^{2}\right)=M \frac{d}{d M} g^{2}(M)=\frac{3 C(G)}{8 \pi^{2}}\left[m^{4} \mathscr{H}_{C C}\right]^{-1}=-\frac{g^{4}}{8 \pi^{2}} \frac{3 C(G)}{1-\frac{1}{8 \pi^{2}} C(G) g^{2}}
$$

with $g^{2}(M)=C / m^{2}$. Hence, the NSVZ $\beta$ function (5.23) [20], with its two coefficients, follows from the matching of the $U(1)_{\widetilde{R}}$ and dilatation anomalies and, in the Wilson effective lagrangian, from the one-loop running which defines the Wilson coupling $g_{W}^{2}$. The important point is the existence of two gauge-invariant superfields, the chiral $\widetilde{\operatorname{Tr}} \mathscr{W} \mathscr{W}$, as usual, and the real $L-2 \Omega$ which appears when the linear superfield is used as gauge coupling field. These two superfields are in natural relation with the two anomaly superfields $X$ and $\chi_{\alpha}$ of the supercurrent structure. Notice also that holomorphicity is entirely absent in this discussion of $\mathscr{N}=1$ super-Yang-Mills, in contrast with $\mathscr{N}=2$ with its Kähler scalar manifold. A similar line of reasoning can be followed to derive the effective action for gaugino condensation, using both counterterms (5.13) and (5.19). ${ }^{34}$

In simple string compactifications to four dimensions with $\mathscr{N}=1$ supersymmetry, the linear multiplet $L$ describes the dilaton and is then, naturally, the gauge coupling and loop-counting superfield [17]. Its role in anomaly cancellation of Kähler anomalies [24] and in particular in the derivation of heterotic gauge threshold corrections [25] has been established long ago. As a sequel, in the framework of conformal supergravity, a (somewhat obscure) derivation from anomalies of the NSVZ $\beta$ function with the linear gauge coupling field has already been given in ref. [26]. In this approach, the renormalization-group behaviour is the response of the theory to a rescaling of the compensating field for dilatation symmetry [27, 28]. This section proposes a derivation in the simpler framework of global $\mathscr{N}=1$ supersymmetry, based on similar arguments and using supercurrent structures.

With constant gauge coupling, the all-order results (5.21) have been obtained by Shifman and Vainshtein [18]. The importance and a calculation of the residual anomaly (5.17) for the NSVZ $\beta$ function has been given with much clarity by Arkani-Hamed and Murayama [29].

Strictly speaking, theory (5.4) does not have an axion: the helicity zero superpartner of $C$ is the antisymmetric tensor $B_{\mu \nu}$ with gauge invariance $\delta B_{\mu \nu}=\partial_{\mu} \Lambda_{\nu}-\partial_{\nu} \Lambda_{\mu}$ and $L$ only includes the gauge-invariant curl

$$
H_{\mu v \rho}=3 \partial_{[\mu} B_{v \rho]}-\omega_{\mu v \rho} .
$$

The antisymmetric tensor couples to the gauge Chern-Simons form $\omega_{\mu v \rho}$ and the effective lagrangian does not have a perturbative dependence on a vacuum angle even if the gauge coupling

\footnotetext{
${ }^{34}$ See refs. [23, 7].
} 
has an all-order expansion. The antisymmetric tensor is dual to a pseudoscalar $\sigma$ with axionic shift symmetry and universal coupling

$$
\frac{1}{4} \sigma \widetilde{\operatorname{Tr}} F_{\mu v} \widetilde{F}^{\mu v}
$$

for all functions $\mathscr{H}$ : the quantum corrections to $\mathscr{H}$ appear in the kinetic lagrangian

$$
-\left(\mathscr{H}_{C C}\right)^{-1}\left(\partial_{\mu} \sigma\right)\left(\partial^{\mu} \sigma\right) .
$$

It is admissible to work with $C$ and $\sigma$ but the resulting chiral supermultiplet is not in a Kähler basis and supersymmetry variations explicitly depend on the function $\mathscr{H}$ defining the lagrangian. With $\mathscr{H}$ as given in expression (5.20), the Legendre transformation turning $C$ into the standard superpartner of $\sigma$ in a kählerian chiral multiplet cannot be analytically solved and information would be lost in an approximate treatment.

\section{Acknowledgements}

I wish to thank the organizers of a brilliant Planck conference at Ioannina University, Jelle Hartong and, at earlier stages of this work, Nicola Ambrosetti and Daniel Arnold for collaboration. Parts of my work has been performed during several stays at the Ecole Normale Supérieure, Paris, the Ecole Polytechnique, Palaiseau and the NCTS at National Tsing Hua University, Hsinchu, Taiwan. Hospitality and discussions with many colleagues are gratefully acknowledged.

\section{References}

[1] C. G. Callan, Jr., S. R. Coleman, R. Jackiw, Annals Phys. 59 (1970) 42-73.

[2] S. R. Coleman and R. Jackiw, Annals Phys. 67 (1971) 552.

[3] S. Coleman, Aspects of Symmetry, Cambridge University Press, Cambridge, 1988, 402 pages. Chapter 3 .

[4] J. Polchinski, Nucl. Phys. B 303 (1988) 226.

[5] S. Ferrara and B. Zumino, Nucl. Phys. B 87 (1975) 207.

[6] D. Arnold, J.-P. Derendinger and J. Hartong, Nucl. Phys. B 867 (2013) 370 [arXiv:1208.1648 [hep-th]].

[7] N. Ambrosetti, D. Arnold, J.-P.-Derendinger and J. Hartong, to appear.

[8] Z. Komargodski and N. Seiberg, JHEP 1007, 017 (2010) [arXiv:1002.2228 [hep-th]].

[9] T. E. Clark, O. Piguet and K. Sibold, Nucl. Phys. B 143 (1978) 445.

[10] M. Magro, I. Sachs and S. Wolf, Annals Phys. 298 (2002) 123 [hep-th/0110131].

[11] S. M. Kuzenko, JHEP 1004 (2010) 022 [arXiv: 1002.4932 [hep-th]]; Eur. Phys. J. C 71 (2011) 1513 [arXiv:1008.1877 [hep-th]].

[12] M. Kaku, P. K. Townsend and P. van Nieuwenhuizen, Phys. Rev. D 17 (1978) 3179; M. Kaku and P. K. Townsend, Phys. Lett. B 76 (1978) 54. 
[13] T. Kugo and S. Uehara, Nucl. Phys. B 226 (1983) 49; Nucl. Phys. B 222 (1983) 125.

[14] S. Ferrara, L. Girardello, T. Kugo and A. Van Proeyen, Nucl. Phys. B 223 (1983) 191.

[15] S. Ferrara and P. van Nieuwenhuizen, Phys. Lett. B 74 (1978) 333; Phys. Lett. B 76 (1978) 404; K. S. Stelle and P. C. West, Phys. Lett. B 74 (1978) 330; Phys. Lett. B 77 (1978) 376.

[16] M. F. Sohnius and P. C. West, Phys. Lett. B 105 (1981) 353.

[17] S. Cecotti, S. Ferrara and M. Villasante, Int. J. Mod. Phys. A 2 (1987) 1839.

[18] M. A. Shifman and A. I. Vainshtein, Nucl. Phys. B 359 (1991) 571.

[19] M. A. Shifman and A. I. Vainshtein, Nucl. Phys. B 277 (1986) 456.

[20] V. A. Novikov, M. A. Shifman, A. I. Vainshtein and V. I. Zakharov, Nucl. Phys. B 229 (1983) 381; B 229 (1983) 407; Phys. Lett. B 166 (1986) 329.

[21] D. R. T. Jones, Phys. Lett. B 123 (1983) 45.

[22] G. Veneziano and S. Yankielowicz, Phys. Lett. B 113 (1982) 231.

[23] C. P. Burgess, J.-P. Derendinger, F. Quevedo and M. Quiros, Phys. Lett. B 348 (1995) 428 [hep-th/9501065].

[24] G. Lopes Cardoso and B. A. Ovrut, Nucl. Phys. B 369 (1992) 351.

[25] J. P. Derendinger, S. Ferrara, C. Kounnas and F. Zwirner, Nucl. Phys. B 372 (1992) 145.

[26] J. -P. Derendinger, S. Ferrara, C. Kounnas and F. Zwirner, Phys. Lett. B 271 (1991) 307.

[27] M. T. Grisaru and P. C. West, Nucl. Phys. B 254 (1985) 249.

[28] S. J. Gates, M. T. Grisaru, M. Rocek and W. Siegel, Superspace Or One Thousand and One Lessons in Supersymmetry, Front. Phys. 58 (1983) 1 [hep-th/0108200].

[29] N. Arkani-Hamed and H. Murayama, JHEP 0006 (2000) 030 [hep-th/9707133]. 